\title{
Bases para el estudio de la adquisición del sistema verbal del español como lengua extranjera
}

\author{
José M. Bustos GisBerT \\ Universidad de Salamanca \\ jbustos@usal.es
}

\begin{abstract}
RESUMEN
Los trabajos sobre la adquisición del sistema verbal del español despiertan dudas referidas al número de aprendices que configuran la base del estudio, a la valoración del grado de conocimiento de nuestra lengua por parte de los estudiantes que configuran el análisis, y a la muestra de lengua y la clase de prueba empleadas para evaluar el uso. Hemos de dotarnos de un procedimiento científico sustentado en la existencia de una base conceptual sólida y en la puesta en marcha de un análisis coherente y consistente de los datos recabados. La base científica para la construcción de muestras de trabajo fiables se encuentra en la estadística inferencial: debemos concretar nuestros estudios en universos de trabajo accesibles y aprehensibles. Para ello buscaremos los aprendices de español en áreas donde estén adecuadamente cuantificados. El espacio de trabajo ideal serán las pruebas de evaluación de lenguas existentes en la actualidad basadas en escalas de niveles que cuenten con un alto grado de fiabilidad.
\end{abstract}

Palabras clave: adquisición de segundas lenguas, evaluación, escalas de nivel, estadística inferencial.

\begin{abstract}
Research into Spanish verb acquisition raises considerable doubts with regard to the number of learners forming the basis of the study, the knowledge of Spanish those learners have, and the type of language and tests applied to assess their use of the language. It is essential that we adopt a scientific method based on inferential statistics, which will provide us with a solid conceptual basis, and coherent and consistent data analysis. To put this method into practice, we must focus our research on accessible and attainable working universes, and, therefore, work with learners of Spanish in areas where they can be properly quantified. Language assessment tests based on level scales and with a high degree of reliability are the ideal workspace.
\end{abstract}

Keywords: Second Language Acquisition, Testing, Level Scales, Inferential Statistics.

Sumario: 1. Introducción, 2. El tamaño de la muestra, 3. Escalas de niveles y pruebas de evaluación como base de los estudios de adquisición, 4. El nivel de los informantes, 5. Los datos y el tipo de prueba usados para conseguirlos. 6. Conclusión 


\section{Introducción}

Los esfuerzos de la investigación vinculada a la adquisición del sistema verbal en el marco del español como lengua extranjera (ELE) se han orientado al menos en dos grandes direcciones. En primer lugar están los trabajos que han focalizado su interés en el planteamiento de estudios contrastivos entre los paradigmas verbales de diferentes lenguas; en segundo lugar se encuentran los estudios de quienes han concentrado su atención en interpretar la adquisición del sistema verbal del español.

Efectivamente, no son pocas las investigaciones que dentro y fuera de nuestras fronteras se han dedicado a comparar los sistemas verbales entre las distintas lenguas. Dejamos al margen ahora los esfuerzos que hasta la fecha se han realizado en la redacción de gramáticas contrastivas para fijarnos en trabajos exclusivamente dedicados al sistema verbal ${ }^{1}$. Sin ánimo de ser exhaustivos, merece la pena recordar ahora algunos trabajos, que resultan muy numerosos en el caso del inglés y el español. Así, Fernández (1990) y Zamorano (2006) han realizado análisis globales del sistema verbal en ambas lenguas; Ruiz y Guijarro (2005) se han ocupado de las diferencias tempo aspectuales derivadas del uso del imperfecto y el indefinido; por su parte, Mansilla (1980) se ha fijado en el presente; la expresión del futuro ha sido estudiada por Estapá y Mott (1989); Bellido (1989) se ha centrado en la voz pasiva; Casorrán (1992) se ha detenido en la modalidad verbal; y García Parejo y Ambandiang (1999) han analizado las diferencias y similitudes desde una perspectiva morfológica.

Por lo que toca al alemán, Cartagena (1994) y después Sánchez Prieto (2003a 2003b, 2004) han aportado un exhaustivo contraste de los sistemas verbales; por su parte Schifko (1967) y Medina (1979) han comparado el subjuntivo; Gauger (1980) se ha centrado en la voz pasiva; por último recordamos el trabajo de Cartagena (1992) dedicado al futuro.

La tercera lengua más comparada es el francés. Dubsky (1961) se ocupa del aspecto verbal, Tresaco (1982) contrasta el sistema verbal de forma global, Eggs (1993) se fija los tiempos pasados y Camps (2002) analiza la traducción de la temporalidad.

Finalmente debemos citar una serie de trabajos referidos a otras lenguas: Tirado y Herrador (2000) analizan las expresiones aspectuales en español y ruso; Leontaridi (2001 y 2002) se fija en las diferencias generales con el griego como punto de referencia; Markic (1991 y 2001) se ocupa de la expresión tempoaspectual en

${ }^{1}$ Solé \& Solé (1977), Whitley (1986) o Hill y Bradford (1991) han elaborado gramáticas contrastivas para el inglés y el español. En el caso del alemán, véase Cartagena y Gauger (1989). Para el portugués, recordemos Moreno y Eres (2007). Para el árabe, Hussein (2004). Moreno y Eres (2007) escriben para lusohablantes de Brasil y aportan una gramática contrastiva con algunos apuntes interesantes en lo que afecta a la temporalidad. Es, no obstante, una gramática didáctica pensada para estudiantes de nivel B2 y C1. También para lusohablantes, Gutiérrez Quintana (2006b). Para el italiano, Gutiérrez Quintana (2006a). 
esloveno y en castellano; Zhou (1995) dedica parte de su trabajo a las divergencias en este ámbito entre español y chino; y en el caso del italiano, Muñiz (1984) compara el condicional y Burr (1989) se centra en el sistema verbal en el uso cotidiano.

Algunos trabajos importantes establecen comparaciones entre más de dos lenguas. Así, Wu de Zierer (1965) se centra en el gerundio en español, inglés, francés y alemán; Brill (1984) se ocupa de los tiempos pasados en francés, italiano y español; Zimmer (1992) estudia la morfología verbal en español, italiano y portugués; Bertinetto y Delfitto (1996) analizan la expresión de la progresividad en italiano, inglés y español; y Martínez-Atienza (2006) compara el sistema tempoaspectual en español, inglés e italiano.

Por lo que toca a la segunda de las vías de investigación, también la producción es copiosa. Esta se plantea en dos grandes direcciones. En la primera, cabe citar estudios generales sobre adquisición que incluyen capítulos específicamente dedicados a la adquisición del sistema verbal del español. Así, contamos con trabajos como Martin González (1999), centrado en hablantes de checo; Torijano (2002) estudia a luso hablantes; Sánchez Iglesias (2003) y Gutiérrez Quintana (2003) trabajan con italohablantes; y Alexopulu (2006) lo hace con griegos. No podemos terminar estas líneas sin citar dos trabajos clásicos y fundamentales en los estudios de adquisición en nuestro país, como son el de Santos Gargallo (1993), centrado en hablantes de serbo croata, y el de Fernández (1997) que se ocupa de la adquisición en hablantes de diversas procedencias lingüísticas ${ }^{2}$.

En la segunda dirección transitan los trabajos centrados en la adquisición del sistema verbal. Dentro de esta opción cabe distinguir los estudios generales centrados en la adquisición de aquellos que focalizan el análisis desde un grupo de aprendices que comparten, aunque no siempre, la misma lengua materna ${ }^{3}$. Estos últimos son los más numerosos. No nos podemos detener en todos, razón por la cual hemos seleccionado aquellos que se consideran actualmente de referencian en la disciplina que nos ocupa. Podemos resumirlos de la siguiente manera:

${ }^{2}$ Cabe recordar que también Sossouvi (2003) estudia la adquisición de ELE en estudiantes benineses bilingües o multilingües.

${ }^{3}$ Por lo que toca a los primeros, es necesario apuntar los trabajos de Baralo (2006 y 2007). 


\begin{tabular}{lll}
\hline Objeto del estudio & Autor(a) & L1 de los aprendices \\
\hline Expresión del pasado & Sánchez Castro (2008) & Alemán \\
\hline Adquisición del subjuntivo & Stokes (1988) & Inglés \\
& Collentine (1995) & Inglés \\
& Lubbers Quesada (1998) & Inglés \\
& Gudmestad (2006) & Inglés \\
& Di Franco (2007) & Italiano \\
\hline \multirow{2}{*}{$\begin{array}{l}\text { Adquisición de las diferencias } \\
\text { tempo aspectuales en el uso } \\
\text { de imperfecto e indefinido }\end{array}$} & Ti Chau (1975) & Inglés \\
& Barnwell (1987) & Inglés \\
& Ramsey (1990) & Inglés \\
& Hasbún (1995) & Inglés \\
& Lafford (1996) & Inglés \\
& Güell (1998) & Varias lenguas \\
& Salaberry (1999) & Inglés \\
& Montrul y Slabakova (1999) & Inglés \\
& Cadierno (2000) & Danés \\
& Camps (2000 y 2002) & Inglés \\
& Díaz y Bekiou (2006) & Varias lenguas \\
& Cuza Blanco (2008) & Inglés \\
& Mao (2009) & Chino mandarín \\
\hline
\end{tabular}

Cuadro 1. Estudios sobre la adquisición del sistema verbal español

Estos estudios relativos a la adquisición del sistema verbal se caracterizan por su enorme heterogeneidad en el proceso investigador y por la escasez de consenso en los resultados alcanzados por los diferentes especialistas que se han dedicado a la cuestión. Se detecta una enorme variedad de métodos de análisis que dificulta a su vez la integración de las conclusiones a las que unos y otros llegan. Es nuestro objetivo en este trabajo reunir en un solo texto los diferentes proyectos puestos en marcha para realizar una revisión crítica en lo que tiene que ver con los planteamientos metodológicos que a ellos subyacen.

Tales trabajos emanan de una fundamentación metodológica que necesita de manera urgente de una profunda reflexión con el objeto de rediseñar herramientas de las cuales puedan derivar resultados consistentes y fiables. Resultados que, a fin de cuentas, sirvan de base para su aplicación directa en la labor subsiguiente y a la que nuestra investigación ha de focalizar su energía: la mejora de los modelos didácticos aplicados en la enseñanza de nuestra lengua. En palabras de Lafford (2000, p. 73), "besides serving as a resource for classroom teachers, future SLA research needs to rely on knowledge of what goes on in the FL classroom in order to test hypotheses and refine its theories. Savignon $(1990,197)$ notes that in addition to relying on natural Second Language Acquisition data, SLA theory needs to be informed by studies of classroom language acquisition."

Un análisis a fondo de los trabajos que hemos encontrado nos permite elevar algunas reflexiones que nos parece oportuno compartir con los interesados en esta 
materia. Se nos despiertan dudas que afectan en buena medida a la validez de muchos de los esfuerzos realizados. Y es que cualquier investigación científica que se precie debe sustentarse en dos grandes pilares: la existencia de una base conceptual sólida y la puesta en marcha de un procedimiento de análisis coherente y consistente.

Un trabajo como el que ahora proponemos difícilmente puede dar respuesta a las dos cuestiones que apuntamos. Especialmente en lo que afecta a la primera de ellas; esto es, al sustrato teórico sobre el que se apoya la investigación adquisicionista. Recordamos ahora, y solo a modo de ejemplo, el trabajo de Güell (1998), quien realiza un completo recorrido por las diferentes perspectivas que en ese sentido se pueden asumir como punto de partida. Sin que ello implique que compartamos su perspectiva de análisis: esa es una cuestión que dejamos para otro momento.

Y lo hacemos por una razón: más allá del propio punto de partida epistemológico, la investigación se resiente en su credibilidad si la metodología de análisis no se sustenta en bases consistentes. Los trabajos que hemos recordado en las páginas precedentes nos despiertan dudas. Dudas que tienen que ver con, al menos, las siguientes consideraciones: el número de aprendices de ELE que configuran la base del estudio, la valoración del grado de conocimiento de nuestra lengua por parte de los estudiantes que sirven de base para el estudio, y la muestra de lengua y el tipo de prueba empleada para evaluar el uso del contenido en cuestión. A estas tres cuestiones queremos dedicar este trabajo.

\section{El tamaño de la muestra}

El punto de partida para la elaboración de esta parte de nuestro trabajo está en las reflexiones de Ellis (1994, p. 49) a la hora de proponer condiciones de fiabilidad y validez para los estudios realizados en el campo del análisis de errores. Allí dedica un capítulo importante a la delicada cuestión de cómo seleccionar el corpus de estudio:

We can identify three broad types of EA according to the size of the sample. A massive sample involves collecting several samples of language use from a large number of learners in order to compile a comprehensive list of errors, representative of the entire population. A specific sample consists of one sample of language use collected from a limited number of learners, while an incidental sample involves only one sample of language use produced by a single learner. Clearly an EA based on a massive sample is a major undertaking and it is not surprising that most published essays have employed specific or incidental samples.

Necesitamos dar un paso más allá en relación con lo que ahora nos ocupa. En el estudio de la adquisición de ELE, hemos de dotarnos de un procedimiento científico en el sentido estricto del término. Beltrán (2003) propone un excelente modelo investigador que, previsto inicialmente para la sociología, es perfectamente asumible como sustento para los estudios de adquisición: en su percepción, la complejidad del objeto de estudio impide que exista un modelo único de análisis, por lo que han de combinarse al menos tres perspectivas. 
La primera es de corte comparativo: debemos acumular una serie de datos con el objeto de establecer diferencias entre procesos paralelos, entre dos o más realidades que comparten rasgos comunes. Así, nosotros deberíamos plantear diferencias entre la adquisición en L1 y en L2, entre la actuación en L1 y en L2; pero también entre las diferentes interlenguas (en adelante IL) que se detectan en el proceso de aprendizaje. Para comparar se hace imprescindible que existan grados mínimos de analogía estructural: siguiendo a Beltrán, la comparación es relevante tanto por las diferencias como por las semejanzas. Por todo ello, habrá que postular la realización de estudios adquisicionistas que respeten esa citada analogía al menos en lo que afecta a dos grandes variables: la lengua materna de los aprendices y su grado de dominio de la L2. Sólo desde estos dos puntos de referencia, podremos establecer poblaciones de análisis fiables.

La segunda propone la asunción de un método crítico racional: no se trata solamente de describir la realidad, sino también de interpretarla, de discutirla y eventualmente de proponer alternativas. La ciencia social y humana no es un ejercicio positivista libre de valoraciones. Se trata, al final, de ir más allá de los hechos a través de un ejercicio de reflexión racional. Todo ello no supone en ningún caso que debamos defender dogmáticamente concepto alguno, ni tampoco implica que tengamos que necesariamente coincidir en los juicios. Significa, ni más ni menos, que no debemos limitarnos a describir el proceso de adquisición de ELE, sino que debemos buscar las causas que explican el desarrollo en una u otra dirección $y$, eventualmente, hemos de realizar propuestas de naturaleza didáctica que nos permitan intervenir en el mencionado proceso.

La tercera aboga por el uso de procedimientos cuantitativos y cualitativos en el análisis. Las ciencias humanas y sociales pueden y deben utilizar mediciones matemáticas puesto que la sociedad lleva a cabo actividades donde el aumento o la reducción cuantitativa es más que relevante: pero la cantidad, por importante que sea, nunca es la razón o la explicación. El número se ha de explicar y analizar de manera crítica y racional. El procedimiento cuantitativo es un paso en el proceso de análisis, no el fin en sí mismo. Ibáñez (1979) defiende que no existe preeminencia entre lo cuantitativo y lo cualitativo, sino que más bien se ha de hablar de concurrencia, donde uno no existe sin el otro. La realización de estudios cuantitativos y cualitativos en el campo de la adquisición de ELE nos obliga a dar respuesta consistente a algunas cuestiones fundamentales, como son el tamaño y la naturaleza de la muestra de aprendices en los que focalizaremos nuestra atención, de un lado, y el procedimiento de análisis que vamos a aplicar a esos datos, de otro.

Los trabajos sobre adquisición que hemos analizado realizan su investigación sobre la base de muestras de estudiantes que quieren ser representativas de los aprendices de ELE con el fin de realizar algún tipo de extrapolación sobre la base de los resultados alcanzados. Sin embargo, son extraordinariamente heterogéneos en lo que se refiere al número de informantes que se consideran en el estudio. ¿Son esas muestras lo suficientemente fiables? En el cuadro siguiente resumimos los datos con los que contamos: 


\begin{tabular}{lll}
\hline Estudio & Muestra & Detalles de la muestra \\
\hline Ti Chau (1975) & 149 & Estudiantes de secundaria \\
Barnwell (1987) & 85 & Universitarios \\
Stokes (1988) & 27 & 17 han estudiado el extranjero y 10 no \\
Ramsey (1990) & 30 & Estudiantes de diversa procedencia \\
Collentine (1995) & 38 & Universitarios \\
Hasbún (1995) & 80 & Universitarios \\
Lafford (1996) & 13 & Universitarios \\
Güell (1998) & 86 & Universitarios \\
Salaberry (1999) & 16 & 16 universitarios. De ellos 4 “casi nativos" \\
Montrul y Slabakova (1999) & 71 & Aporta datos sobre la edad media de uso y otros \\
Cadierno (2000) & 10 & Universitarios \\
Camps (2000 y 2002) & 30 & \\
Díaz y Bekiou (2006) & 31 & Universitarios \\
Gudmestad (2006) & 37 & Universitarios \\
Di Franco (2007) & 16 & Universitarios \\
Sánchez Castro (2008) & 35 & Universitarios \\
Cuza Blanco (2008) & 20 & Estudiantes avanzados e inmigrantes de larga \\
& & estancia en Canadá \\
Mao, P. (2009) & 93 & Universitarios \\
\hline
\end{tabular}

Cuadro 2. Tamaños de las muestras usadas

La base científica para la construcción de muestras fiables se encuentra en la estadística inferencial ${ }^{4}$. Este modelo de análisis persigue obtener generalizaciones sobre una población determinada a partir del estudio de una muestra de dicha población. La validez de las estimaciones alcanzadas se sustenta esencialmente en la teoría de las probabilidades. El sentido del estudio basado en muestras es evidente: reduce costes materiales, los datos se obtienen a mayor velocidad y los resultados resultan más comprensibles. Los estudios de estadística inferencial ponen especial atención en cómo seleccionar una muestra que proporcione un grado elevado de fiabilidad al estudio del que se trate. Para decidir la muestra y con el objetivo de que no resulte sesgada, se hace necesario tomar en consideración una serie de cuestiones esenciales.

La primera tiene que ver con que toda muestra ha de ser representativa de un universo o población objeto del estudio. Evidentemente, aquí empiezan nuestros problemas. En principio nuestro universo de estudio estaría compuesto por los aprendices de ELE. Pero claro, ¿cómo determinamos sus componentes? ¿Sobre qué variables podremos tomar nuestras decisiones? La respuesta no es en absoluto sencilla, aunque sí crucial para establecer puntos de partida científicos que resulten consistentes. Y no lo es, entre otras cosas, porque nos encontramos con dificultades en la contabilización misma de nuestro universo de trabajo. ¿Cuántos estudiantes de

\footnotetext{
${ }^{4}$ Seguimos en lo esencial a García Ferrando (2000) y a Rodríguez Osuna (2003).
} 
ELE podemos reunir? ¿Cómo lo hacemos? ¿Por lenguas? ¿Por nacionalidades? ¿Por niveles de aprendizaje?

Los datos que podemos manejar al respecto no son en absoluto homogéneos ni fiables. Citaremos a modo de ejemplo tres publicaciones diferentes y haremos alguna referencia específica a los datos proporcionados para el caso de Estados Unidos, por ser uno de los países donde reside un mayor número de aprendices de nuestro idioma.

En 2006 el Instituto Cervantes edita la Enciclopedia de Español en el mundo. En ella se estima que el número de estudiantes e ELE es de 11,3 millones aunque luego se redondea la cifra en nada menos que 14 millones $^{5}$. En esa estimación se mezclan datos desde 2003 a 2006. Este es el texto que encabeza el trabajo:

Al menos 14 millones de alumnos estudian español como lengua extranjera en el mundo. Éste es el resultado de sumar el número de estudiantes de español existentes en la actualidad en 86 países que no son de lengua española, y cuyos datos pormenorizados se ofrecen en la tabla que va a continuación. Las cifras se refieren a todos los niveles de la enseñanza - incluida la no reglada - y se ciñen a los datos disponibles en cada uno de los países. Esos datos no siempre son completos ni exhaustivos, y en ocasiones no ha sido posible recabar cifras concretas y actualizadas en determinados ámbitos educativos, como en el caso de los centros de enseñanza privada, reacios en su mayoría a aportar datos oficiales en cuanto a los estudiantes que demandan sus cursos de español. Por ello, el Instituto Cervantes ha calculado que la demanda real de español es, como mínimo, superior en un $25 \%$ a los datos que aquí se ofrecen.

De ellos se atribuyen 6 millones a Estados Unidos. Pues bien, en 2008, el mismo Instituto Cervantes publica su Enciclopedia del Español en los Estados Unidos. Allí aporta una cifra que no llega a 5 millones de estudiantes entre la enseñanza secundaria y la universitaria. En otras palabras, si el propio Instituto Cervantes solo se atreve a proponer cifras con un margen de error de $\pm 25 \%$ para el cómputo total de aprendices de ELE. Y si ese margen del error es de $\pm 15 \%$ para el caso de Estados Unidos, todo ello significa que no podemos construir un universo de estudio válido sobre la base de los datos con lo que en la actualidad contamos y que se refieren a la población completa de aprendices de ELE.

${ }^{5}$ Con el problema añadido de que esa cifra no incluye los estudiantes de ELE dentro de España, lo que introduce una variable más que no podemos tener en cuenta. Carrera y Gómez Asencio (2009) nos recuerdan que existen en la actualidad en España aproximadamente 600 centros de enseñanza de ELE. La Secretaría de Estado de Turismo hizo una estimación de 130.000 alumnos de ELE en España para 2001 (Carrera y G. Asencio, 2009, p.22). Esa cifra coincide básicamente con la de la Base de Datos del Instituto Cervantes para 2006, donde cuantifica unos 116.000 alumnos. Por su parte, Berdugo (2006) propone 182.000 alumnos para 2004 y 192.000 para 2005 (Carrera y G. Asencio, 2009, p.23). Y todo ello sin contar a los más de 25.000 estudiantes Erasmus que cada año pasan por las aulas universitarias de nuestro país. 
Podemos comparar los datos del Instituto Cervantes con los que nos ofrece en Ministerio de Educación para los Estados Unidos en sus informes El Mundo estudia español correspondientes a 2005, 2006 y 2007. Son exactamente iguales en los tres anuarios. El resultado es que se puede hablar de aproximadamente 4.750.000 estudiantes, cifra que se acerca más a la incluida en la segunda de las publicaciones del Instituto Cervantes, pero que se aleja de los hipotéticos seis millones que presumía en $2006^{6}$.

Los datos con los que contamos nos pueden dar, efectivamente, una buena imagen acerca de la importancia global de la enseñanza de ELE en el mundo. Sin embargo, las cifras no son en absoluto consistentes a la hora de efectuar un análisis estadístico fiable.

Podríamos entonces optar por realizar nuestra labor sobre la base de muestreos no aleatorios, que no necesitan de una identificación del universo de trabajo ni una selección basada en el cálculo de probabilidades ${ }^{7}$. De hecho, los trabajos que hemos seleccionado como punto de partida son de esta naturaleza ${ }^{8}$. El problema fundamental es que no se puede saber si en términos de investigación son representativos de la realidad. En palabras de Ferrando (2000), no permiten estimar los márgenes de error, razón por la cual se debe evitar su uso.

En nuestro universo general de trabajo, la población teórica que deberíamos estudiar no es accesible porque no está cuantificada y censada. Pero ello no significa que no podamos realizar investigaciones estadísticamente convincentes, sino que debemos concretar ese total en lo que se denomina un universo de trabajo accesible y aprehensible. ¿Cómo? Buscando áreas donde los aprendices de ELE estén adecuadamente cuantificados. ¿Dónde? En los pruebas de evaluación de lenguas existentes en la actualidad basadas en escalas de niveles que cuenten con un alto grado de fiabilidad.

Para concluir y solo por apuntar ahora algunos datos relevantes, recordemos que en los estudios estadísticos inferenciales se asume que, cuanto mayor sea el número de individuos que se incluyan en la muestra, más seguro se estará de los resultados. Las estimaciones realizadas sobre muestras muy pequeñas, tal y como ocurre en los trabajos que justifican nuestras investigación, tienen un grado de desviación muy elevado. ¿De qué depende el tamaño de la muestra? De la varianza poblacional, del

${ }^{6} \mathrm{Y}$ no perdamos de vista que son datos con ya cierta antigüedad, puesto que de facto corresponden a 2002.

${ }^{7}$ Nos estamos refiriendo a los modelos basados en muestras accidentales, muestras de cuota $o$ en muestras intencionadas. Las primeras usan individuos elegidos por simple proximidad con el investigador; las segundas, muy utilizadas por los analistas de los medios de comunicación, se basan en que se construye el perfil del individuo buscado y el investigador lo elige libremente de entre los miembros del grupo; en las terceras se asume que el sentido común del investigador le permite incluir adecuadamente la muestras sobre la base normalmente de criterios subjetivos.

${ }^{8}$ Se trata de muestreos no probabilísticos efectuados sobre la base de la imposibilidad de fijar adecuadamente el universo de trabajo. 
margen de error asumible y del nivel de confianza exigible ${ }^{9}$. Afortunadamente, se cuenta ya con tablas para determinar el tamaño de la muestra basadas en un nivel de confianza del 95\% y con unos márgenes de error que van del 1 al 10\%. Reproducimos la que nos propone Ferrando $(2000)^{10}$ :

Tabla para la determinación de una muestra sacada de una población finita, para márgenes de error de 1, 2, 3, 4, 5, 10 por 100 , en la hipótesis de $p=50$ por 100. Nivel de confianza del 95,5 por 100

\begin{tabular}{|c|c|c|c|c|c|c|}
\hline \multirow{2}{*}{$\begin{array}{c}\text { Amplitud de la } \\
\text { población }\end{array}$} & \multicolumn{6}{|c|}{ Amplitud de la muestra para margenes de error abajo indicados } \\
\hline & $\pm 1 \%$ & $\pm 2 \%$ & $\pm 3 \%$ & $\pm 4 \%$ & $\pm 5 \%$ & $\pm 10 \%$ \\
\hline 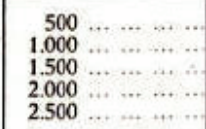 & & 1.250 & $\begin{array}{l}638 \\
714 \\
769\end{array}$ & $\begin{array}{l}385 \\
441 \\
476 \\
500\end{array}$ & $\begin{array}{l}222 \\
286 \\
316 \\
333 \\
345\end{array}$ & $\begin{array}{l}94 \\
83 \\
91 \\
95 \\
96\end{array}$ \\
\hline 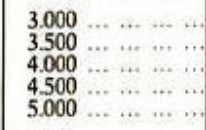 & & $\begin{array}{l}1.364 \\
1.458 \\
1.538 \\
1.607 \\
1.667\end{array}$ & $\begin{array}{l}811 \\
843 \\
870 \\
891 \\
909\end{array}$ & $\begin{array}{l}517 \\
530 \\
541 \\
549 \\
556\end{array}$ & $\begin{array}{l}353 \\
359 \\
364 \\
367 \\
370\end{array}$ & $\begin{array}{l}97 \\
97 \\
98 \\
98 \\
98\end{array}$ \\
\hline 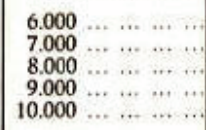 & 5.000 & $\begin{array}{l}1.765 \\
1.842 \\
1.905 \\
1.957 \\
2.000\end{array}$ & $\begin{array}{r}938 \\
949 \\
976 \\
989 \\
1.000\end{array}$ & $\begin{array}{l}566 \\
574 \\
580 \\
584 \\
588\end{array}$ & $\begin{array}{l}375 \\
378 \\
381 \\
383 \\
385\end{array}$ & $\begin{array}{l}98 \\
99 \\
99 \\
99 \\
99\end{array}$ \\
\hline 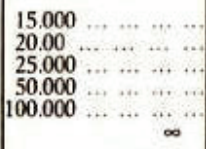 & $\begin{array}{r}6.000 \\
6.667 \\
7.143 \\
8.333 \\
9.091 \\
10.000\end{array}$ & $\begin{array}{l}2.143 \\
2.222 \\
2.273 \\
2.381 \\
2.439 \\
2.500\end{array}$ & $\begin{array}{l}1.034 \\
1.053 \\
1.064 \\
1.087 \\
1.099 \\
1.111\end{array}$ & $\begin{array}{l}600 \\
606 \\
610 \\
617 \\
621 \\
625\end{array}$ & $\begin{array}{l}390 \\
392 \\
394 \\
397 \\
398 \\
400\end{array}$ & $\begin{array}{r}99 \\
100 \\
100 \\
100 \\
100 \\
100\end{array}$ \\
\hline
\end{tabular}

$p=$ proporción (en porcentajes) de los elementos portadores del carácter conside. rado. Si $p$ es < 50 por 100 la muestra necesaria es más pequeña.

Flente: G. Taglacarne, Técnica y práctica de las Investigaciones de Mercado, 1962. página 156

Cuadro 3. Tabla para determinar el tamaño de una muestra

Aún no hemos llegado a cerrar nuestro universo de trabajo, pero la propia tabla nos apunta datos interesantes: los estudios parten de poblaciones de al menos 500 individuos como universo completo; además, en términos absolutos, como mínimo exigen casi 100 individuos de muestra. Ese número apenas se alcanza en una de las investigaciones que nos han servido como punto de partida. Los datos apuntados nos permiten, pues, concluir que los estudios con los que hasta ahora contamos nos

${ }^{9}$ Quizá el concepto menos claro sea el primero: tiene que ver con el grado de dispersión que se pueda identificar en la población de estudio; cuando la población es muy homogénea, la varianza se reduce.

${ }^{10}$ Idéntica tabla se encuentra en Arkin \& Colton (1968), en Bugeda (1974) y en Santos et al. (2004). Parece que en el texto de Ferrando se ha colado una errata en la columna correspondiente al margen de error del $\pm 10 \%$ En el resto de las tablas citadas la serie de número es $83,91,94$ para las tres primeras cifras que aparecen. 
pueden apuntar tendencias o despertar hipótesis enormemente sugerentes, pero en términos estadísticos las muestras no son lo suficientemente representativas como para proporcionar resultados científicamente fiables.

\section{Escalas de niveles y pruebas de avaluación como base de los estudios de adquisición}

Existe abundante bibliografía en la que se establecen espacios comunes de análisis entre las investigaciones vinculadas a adquisición de segundas lenguas (ASL) y las que tienen que ver con la evaluación de lenguas. Cabe citar entre otros los trabajos de Cohen (1998), Brindley (1998), Sohamy (2000) y Douglas (2001) ${ }^{11}$.

Brindley (1998) centra su interés en estudiar la aplicación de las escalas de niveles de conocimiento al campo de la ASL, específicamente de aquellas configuradas desde la perspectiva del comportamiento lingüístico de los aprendices ya que reflejarían etapas del comportamiento lingüístico en una o varias áreas. Estarían, al fin y al cabo, indicando niveles de IL desde cero hasta una expresión cuasi nativa. Son gradaciones destinadas a medir la actuación global sobre la base de una serie de competencias subyacentes. Se trata, pues, de evaluar la competencia comunicativa global de los aprendices desde su competencia lingüística, su competencia expresiva y su competencia comprensiva.

El problema, en opinión de Brindley, puede estar en cómo se construyen las escalas de niveles, en tanto que muchas de ellas, en sus palabras, carecen de fundamentación teórica. En la medida en que sean fiables, también serán aplicables a ASL, como apuntan Griffin y Nix (1991). Debemos, pues, fiarnos, de aquellas que se hayan construido de forma muy consistente y sobre la base de información perfectamente detallada. En ese sentido, y seguimos ahora a North (1993), pueden convertirse en herramientas perfectamente prácticas y operativas en los estudios de ASL. ¿De cuáles podremos fiarnos? Evidentemente, de aquellas con un alto grado de validez. Y este se puede obtener haciendo una valoración que tenga en cuenta al menos las siguientes cuestiones (Brindley, 1998): la relación entre ellas y los resultados en las pruebas de evaluación y acreditación que las reconocen; su validación psicolingüística, en tanto que demuestren que las habilidades descritas para cada nivel son las que efectivamente usan los aprendices para resolver las tareas impuestas en las pruebas de evaluación correspondientes; y la coherencia estructural de la secuencia de niveles propuesta ${ }^{12}$.

En nuestro caso, entendemos que contamos con una escala de niveles perfectamente fiable, como es la que emana del Marco de referencia europeo para el aprendizaje, la enseñanza y la evaluación de lenguas, publicado en 2001 por el

${ }^{11}$ Se sugiere al interesado la lectura del imprescindible trabajo de Bachman y Cohen (1998).

${ }^{12}$ De hecho, en el caso del inglés como lengua extranjera, Brandley duda de la validez de escalas perfectamente conocidas en los Estados Unidos como es el caso de la propuesta por la ACTFL. Dedica una importantísima parte de su trabajo a los estudios existentes para la validación de escalas de nivel. 
Departamento de Política Lingüística del Consejo de Europa. Recordemos el primer párrafo de este documento imprescindible para el contexto de la enseñanza, el aprendizaje y la adquisición de lenguas:

El Marco de referencia europeo proporciona una base común para la elaboración de programas de lenguas, orientaciones curriculares, exámenes, manuales, etc. en toda Europa. Describe de forma integradora lo que tienen que aprender a hacer los estudiantes de lenguas con el fin de utilizar una lengua para comunicarse, así como los conocimientos y destrezas que tienen que desarrollar para poder actuar de manera eficaz. La descripción también comprende el contexto cultural donde se sitúa la lengua. El Marco de referencia define, asimismo, niveles de dominio de la lengua que permiten comprobar el progreso de los alumnos en cada fase del aprendizaje y a lo largo de su vida.

El Marco propone una escala de niveles basada en tres grandes criterios: libre del contexto en tanto que la escala sea aplicable a cualquier itinerario de acceso a la lengua y en la medida en que las categorías de clasificación respondan a lo que efectivamente hacen los aprendices con la lengua; basada en una fundamentación teórica; y libre de conceptos inconsistentes y de cálculos aproximados. Para ello se ha aplicado una metodología que ha incluido procesos intuitivos, cuantitativos y cualitativos $^{13}$.

Cabe recordar ahora que el Marco propone una escala de 6 niveles, esencialmente basados en trabajos previos auspiciados desde el Consejo de Europa. Están establecidos sobre la base de puntos de referencia comunes y descritos a partir de tres grandes categorías: las actividades comunicativas (lo que puede hacer el usuario de la lengua), las estrategias empleadas para llevar a cabo tales actividades y las competencias comunicativas, entendidas desde la triple realidad de competencia lingüística, competencia pragmática y competencia sociolingüística.

Recordaba antes que Brindley (1998) defiende como una prueba de validez de las escalas de niveles la relación que pueda establecerse entre su construcción y los resultados en las pruebas de evaluación y acreditación que las reconocen. No hay duda en que las pruebas de aptitud de lenguas nos proporcionan la información más fiable para la realización de muestras con las que estudiar la adquisición de lenguas extranjeras. En primer lugar porque suponen en términos cuantitativos el corpus de aprendices perfectamente identificado más amplio con el que podemos contar. En segundo lugar porque incluyen aprendices que acceden al idioma por cualquiera de los caminos posibles, estén estos o no vinculados a algún tipo de enseñanza reglada. En tercer término porque nos permiten incluir en la misma muestra a estudiantes que en diferentes momentos del tiempo han exhibido un grado de dominio similar de ELE. En cuarto lugar porque nos posibilitan estratificar la muestra desde al menos

${ }^{13}$ El procedimiento de fijación y definición de la escala es ciertamente complejo a la vez que muy exhaustivo. Se resume, dentro del propio documento del Marco, en los anejos A, B, C y D. 
dos perspectivas, como son la lengua materna y el nivel de dominio en el que los aprendices se encuentran; esto es, desde el tipo de transferencia al que han estado y están sometidos, y desde la IL que en ese momento dominan.

En el caso que nos ocupa, disfrutamos de una correlación muy fuerte entre las escalas de nivel de las que disponemos y los modelos de examen con los que contamos. El Marco dedica un capítulo completo a la cuestión de la evaluación. De hecho, los responsables de su redacción establecen una vinculación evidente entres los contenidos asignados a la escala de niveles y las pruebas de evaluación de lenguas. Así, el Marco recoge en su anejo D sus puntos de comparación con los sistema evaluativos vinculados a la Association of Language Testers in Europe (ALTE). El trabajo de ALTE parte de la definición de los llamados Can Do Statements, desde los que se crea un marco de niveles basado en la actuación lingüística de los usuarios de las L2. En la actualidad el trabajo beneficia ya a 13 lenguas y los resultados se han validado con un procedimiento de autoinformes a los que han respondido en torno a 10.000 aprendices de idiomas de toda Europa.

A continuación se han ubicado los exámenes administrados por los diferentes miembros de ALTE dentro de ese marco a partir de los contenidos de las pruebas, los tipos de tareas y el perfil de los candidatos. Nos referimos ahora a exámenes realizados en 15 lenguas distintas, y a cerca de 70 pruebas de aptitud diferentes ${ }^{14}$. El siguiente paso ha sido relacionar la escala desarrollada en ALTE con otros indicadores de nivel lingüístico. Los propios responsables de la elaboración del Marco han reconocido la validez de la gradación de niveles de ALTE en relación con la propuesta por ellos en ese documento.

En el caso de la acreditación del español como lengua extranjera contamos con un panorama de pruebas bastante completo en el marco de ALTE. Se trata de los exámenes para la obtención de los Diplomas de Español como lengua Extranjera (DELE), otorgados por el Instituto Cervantes en nombre del Ministerio de Educación de España. La Universidad de Salamanca elabora los modelos de examen y evalúa las pruebas para la obtención de los diplomas de español ${ }^{15}$. Las correspondencias serían las siguientes ${ }^{16}$ :

${ }_{15}^{14}$ Pueden consultarse en http://www.alte.org/alteframework/table.php

15 El lector interesado encontrará más información en http://diplomas.cervantes.es/index.jsp

${ }^{16}$ Las pruebas para la obtención del Diploma A1 se realizaron por primera vez en noviembre de 2009. Las pruebas para la obtención del Diploma A2 se comenzaron a realizar en mayo de 2010. En el cuadro se incluyen las denominaciones actuales y sus correspondientes en el pasado. 


\section{Tabla de Correspondencias}

\begin{tabular}{|c|c|c|c|c|c|c|}
\hline $\begin{array}{l}\text { Niveles del } \\
\text { Consejo } \\
\text { de Europa }\end{array}$ & A1 & A2 & B1 & B2 & $\mathrm{C} 1$ & $\mathrm{C} 2$ \\
\hline $\begin{array}{l}\text { Niveles de } \\
\text { ALTE }\end{array}$ & Acceso & Plataforma & Umbral & Avanzado & $\begin{array}{c}\text { Dominio } \\
\text { operativo } \\
\text { eficaz }\end{array}$ & Maestría \\
\hline DELE & $\begin{array}{c}\text { Diploma } \\
\text { de } \\
\text { Español } \\
\text { Nivel A1 }\end{array}$ & $\begin{array}{c}\text { Diploma de } \\
\text { Español } \\
\text { Nivel A2 }\end{array}$ & $\begin{array}{c}\text { Diploma } \\
\text { de } \\
\text { Español } \\
\text { Nivel B1 } \\
\text { (Inicial) } \\
\text { (Antes } \\
\text { C.I.E.) }\end{array}$ & $\begin{array}{c}\text { Diploma de } \\
\text { Español } \\
\text { Nivel B2 } \\
\text { (Intermedio) } \\
\text { (Antes } \\
\text { D.B.E.) }\end{array}$ & & $\begin{array}{c}\text { Diploma } \\
\text { de Español } \\
\text { Nivel C2 } \\
\text { (Superior) } \\
\text { (Antes } \\
\text { D.S.E) }\end{array}$ \\
\hline
\end{tabular}

Cuadro 4. Correspondencias entre el Marco, ALTE y los DELE

En la actualidad, los esfuerzos del Instituto Cervantes y de la universidad de Salamanca se dirigen a que estas correspondencias sean perfectamente válidas. Con todo y con eso, podemos afirmar que contamos con un marco perfectamente fiable para establecer muestras de análisis orientadas a la realización de estudios de adquisición.

\section{El nivel de los informantes}

A continuación debemos referirnos a la cuestión del nivel de ELE de los informantes seleccionados para efectuar los estudios de adquisición. De nuevo hemos de recordar los datos que podemos deducir de las investigaciones que nos sirven como punto de partida. Una vez más las posturas son muy divergentes y las diferencias se establecen en dos grandes direcciones: la primera se refiere al nivel de los individuos que configuran la muestra; la segunda tiene que ver con cómo se ha acreditado tal nivel.

Desde la primera perspectiva se plantean dos grandes posibilidades ${ }^{17}$. Una primera opción restringe el análisis a aprendices de un mismo nivel:

${ }^{17}$ En algunos casos, no se nos proporcionan datos acerca del nivel de ELE de la muestra. Así sucede en los trabajos de de Ti Chau (1975), Cadierno (2000) y Camps (2000 y 2005). 


\begin{tabular}{ll}
\hline Barnwell (1987) & $\begin{array}{l}\text { 85 estudiantes matriculados en el Spanish II en la } \\
\text { Universidad de Pittsburgh. Se encuentran en la mitad del } \\
\text { curso. }\end{array}$ \\
\hline Stokes (1988) & $\begin{array}{l}27 \text { estudiantes de nivel superior matriculados en un } \\
\text { curso de Gramática y Redacción. }\end{array}$ \\
\hline Collentine (1995) & $\begin{array}{l}38 \text { estudiantes de nivel intermedio de la Universidad de } \\
\text { Texas. }\end{array}$ \\
\hline Lubbers Quesada (1998) & $\begin{array}{l}\text { Estudiantes de nivel intermedio en un curso intensivo en } \\
\text { México. }\end{array}$ \\
\hline Di Franco (2007) & $\begin{array}{l}\text { 16 estudiantes de nivel intermedio. } \\
\text { Cuza Blanco (2008) }\end{array}$ \\
$\begin{array}{l}\text { 20 estudiantes cursos avanzados de ELE. No se dan } \\
\text { explicaciones de lo que significa "estudiante avanzado". }\end{array}$ \\
$\begin{array}{l}35 \text { estudiantes que han acabado el primer año de } \\
\text { universidad. }\end{array}$ \\
\hline
\end{tabular}

Cuadro 5. Estudios que analizan aprendices de un mismo nivel

Sin embargo, una opción aún más frecuente mezcla estudiantes de diferentes niveles:

\begin{tabular}{|c|c|}
\hline Ramsey (1990) & $\begin{array}{l}30 \text { estudiantes distribuidos en } 5 \text { niveles. No indica cómo } \\
\text { los distribuye ni cuáles son los niveles. }\end{array}$ \\
\hline Hasbún (1995) & $\begin{array}{l}80 \text { estudiantes distribuidos en } 4 \text { niveles según el curso en } \\
\text { el que están matriculados los estudiantes. No indica } \\
\text { cuáles. }\end{array}$ \\
\hline Lafford (1996) & $\begin{array}{l}13 \text { aprendices correspondientes a tres niveles distintos de } \\
\text { la escala de la ACTFL. No indica cuáles. }\end{array}$ \\
\hline Güell (1998) & $\begin{array}{l}86 \text { estudiantes universitarios extranjeros en Barcelona, } \\
\text { que ubica en } 4 \text { niveles que se corresponden con el Test } \\
\text { SGEL. Indica que los más altos son los avanzados. De } \\
\text { todas maneras reconoce que "el resultado obtenido no } \\
\text { permite clasificarlos claramente en un nivel concreto" } \\
\text { Nivel 1: } 16 \text {. Nivel 2: } 15 \text {. Nivel 3: } 38 \text {. Nivel } 4: 17 \text {. }\end{array}$ \\
\hline $\begin{array}{l}\text { Montrul y Slabakova } \\
\text { (1999) }\end{array}$ & $\begin{array}{l}71 \text { estudiantes intermedios y avanzados de dos } \\
\text { universidades americanas a las que califica como "de } \\
\text { mucha fuerza investigadora" Son } 42 \text { intermedios y } 29 \\
\text { avanzados. No dice cómo fueron elegidos. }\end{array}$ \\
\hline Salaberry (1999) & $\begin{array}{l}16 \text { estudiantes de cuatro cursos diferentes de ELE ( } 112 \text {, } \\
123,203,311) \text { de una universidad estadounidense. }\end{array}$ \\
\hline Díaz y Bekiou (2006) & $\begin{array}{l}31 \text { estudiantes distribuidos en los niveles } 3 \text { y } 5 \\
\text { correspondientes a la escala del Test SGEL. No indican a } \\
\text { qué corresponden. }\end{array}$ \\
\hline Gudmestad (2006) & $\begin{array}{l}37 \text { estudiantes. } 17 \text { de nivel intermedio y } 20 \text { de nivel } \\
\text { avanzado. }\end{array}$ \\
\hline Mao, P. (2009) & 45 estudiantes iniciales y 38 intermedios. \\
\hline
\end{tabular}

Cuadro 6. Estudios que analizan aprendices de diferentes niveles 
Así pues, el panorama no es sencillo. Hemos de tomar decisiones sobre si el nivel de los aprendices que configuran la muestra debe o no ser homogéneo. En cualquier caso, resulta inaceptable no tomar en consideración el nivel puesto que este es correlativo, ya lo hemos dicho antes, con diferentes estados de IL.

Más discutible parece el procedimiento de asignación de nivel a los componentes de la muestra. Obviando los casos en lo que no se indica el nivel y a los que ya nos referimos más arriba ${ }^{18}$, se observa una tendencia a utilizar las nivelaciones de naturaleza administrativa: se presupone que el hecho de que los aprendices estén matriculados en un curso académico, los unifica en un nivel de ELE determinado. De esta discutible percepción parten los trabajos de Barnwell (1987), Stokes (1988), Hasbún (1995), Collentine (1995), Salaberry (1999), Di Franco (2007) y Sánchez Castro (2008). Especialmente llamativo resulta el caso de Gudmestad (2006). Parte de una nivelación administrativa, puesto que elige la muestra a partir del curso que están realizando en la universidad: estudiantes de cuarto semestre de español (a los que interpreta como intermedios) y 20 estudiantes matriculados en un curso de lingüística aplicada en el cuarto año de estudios universitarios, que serían los que valora como estudiantes avanzados. Al objeto de garantizar que la nivelación administrativa es correcta, realiza con ellos una prueba de selección múltiple de 11 items de contenido gramatical: los estudiantes "intermedios" alcanzan unos resultados en los que el que menos acierta tiene 1 respuesta correcta, y el que más, 9. Los "avanzados" se mueven en una franja entre 7 y 11 aciertos. Así y todo, el investigador mantiene inalterables los dos grupos de estudio.

Más allá de las dudas que plantea la prueba elegida para evaluar el nivel de los participantes en la investigación y de la sorpresa que se deriva de ver cómo se continúa con la investigación asumiendo que existen dos grupos diferentes, hay un hecho incuestionable: parece que los aprendices no tienen ese nivel homogéneo que se suponía a partir del curso en el que estaban matriculados. Efectivamente, asumir que un grupo de estudiantes matriculados en el mismo curso disfrutan del mismo nivel de conocimientos sería lo mismo que presuponer que en ese curso todos progresan exactamente de la misma manera. Sinceramente, tal presunción está fuera de la realidad. Por ello, más de un investigador ha querido garantizar la correcta nivelación a partir de algún tipo de prueba. Por este camino se ha podido elegir entre alguna de las tres alternativas que a continuación expondremos.

Una primera opción ha sido utilizar escalas de nivel reconocidas y reconocibles por los investigadores. Es el caso de Lafford (1996), quien ubica a los estudiantes que componen su muestra en tres niveles usando la escala ACTFL OPI ${ }^{19}$ :

Una segunda posibilidad es el uso de pruebas de clasificación para la nivelación de los estudiantes. Es el caso de Lubbers Quesada (1998), quien trabaja con un grupo de aprendices mientras hacen un curso intensivo de español en México para el que

${ }^{18}$ Interesa a hora añadir el estudio de Cuza Blanco (2008), quien reconoce de modo explícito que no hizo ninguna prueba de nivel a los individuos de su muestra.

${ }^{19}$ Otro problema, como indica Brindley (1998) es el grado de validez y fiabilidad de tal prueba. Para conocerla, véase http://www.actfl.org/i4a/pages/index.cfm?pageid=3642 
supuestamente han recibido una nivelación por la vía de una prueba de clasificación. Por otra parte, dentro de nuestras fronteras, dos investigaciones, Güell (1998) y Díaz y Bekiou (2006) utilizan el Test SGEL ${ }^{20}$, una prueba que ya tiene cierta antigüedad. Más allá de esta consideración, las dudas se nos plantean sobre todo por el hecho mismo de tratarse de una prueba de clasificación. En ese sentido, es perfectamente asumible la observación que se realiza en el Diccionario de términos claves de ELE editado por el Centro Virtual Cervantes ${ }^{21}$ :

En didáctica de lenguas, al empleo de un marco de referencia normativo para evaluar aprendizajes, se le suele hacer la crítica siguiente: los resultados obtenidos al comparar la información recogida con la ofrecida por la norma adoptada no informan de si los sujetos evaluados dominan los conocimientos y destrezas que se habían fijado como objetivos que alcanzar, pues no se evalúa el logro del aprendizaje, sino lo que el aprendiente sabe, pero que podía ya saber sin necesidad de intervención didáctica. De este modo, y como reacción al empleo en educación de este tipo de marco de referencia, se plantean otras posibilidades que permitan juzgar la información recogida para evaluar en función del grado de aprendizaje del individuo. La traducción de objetivos en criterios y la comparación con los resultados proporciona un posible punto de referencia denominado criterial.

A esta crítica se añaden otras que se han planteado con frecuencia en el sentido del escaso nivel de fiabilidad de este tipo de pruebas, derivado de las dificultades de administración de las mismas y de las modificaciones que normalmente reciben. La propia inseguridad de los resultados alcanzados, lleva a Güell $(1998$, p. 277) a afirmar lo siguiente:

El resultado obtenido por alguno de los INN (informantes no nativos) no permite clasificarlos claramente en un nivel concreto, sino que, de acuerdo con las tablas establecidas en la prueba SGEL algunos aprendices se sitúan entre dos niveles: 7 INN se clasifican entre los niveles 2 y 3 , y 9 entre los niveles 3 y 4 .

Por ello, decide realizar un nuevo reagrupamiento de la muestra sobre la base del centro de enseñanza al que acuden los informantes durante su estancia en España, para combinar en el estudio ambos criterios clasificatorios.

El tercer camino ha sido la utilización de pruebas propias de nivelación basadas en exámenes que certifican grados de conocimiento. En ambos casos se inspiran parcialmente en pruebas para la obtención de los DELE. Mao (2009) selecciona administrativamente a sus informantes, correspondientes al $2^{\circ}$ y al $5^{\circ}$ curso del Colegio Universitario de Idiomas Wenzao, de Taiwán. Sin embargo, aplican además

${ }^{20}$ Es un test de clasificación estandarizado creado y editado por la editorial SGEL en 1986. Se compone de 100 unidades: 15 de comprensión auditiva y 85 de conocimiento gramatical del español.

$21 \mathrm{http} / /$ cvc.cervantes.es/ensenanza/biblioteca_ele/diccio_ele/diccionario/marcoreferencia evaluacion.htm 
una prueba de gramática y vocabulario correspondiente a exámenes para la obtención de los DELE celebrado en mayo de 2001:

Además, para conseguir una nivelación válida y fiable, además del curso (un factor intergrupo), se realizará un test de nivel en cada grupo. El test que se usa para tal fin está basado en la prueba cuarta de Gramática y Vocabulario del DELE (la convocatoria del 11 de mayo de 2001). Se usa el nivel inicial para los de $2^{\circ}$ y el nivel intermedio para los de $5^{\circ}$, pensando en sus conocimientos lingüísticos ya aprendidos en la institución. La puntuación se considera como un factor intragrupo.

El segundo caso es el de Montrul y Slabakova (1999, p. 21). También parten de una nivelación administrativa. Sin embargo, la validan también con una prueba del DELE.

In order to independently assess the English-speakers' proficiency in Spanish we used a proficiency test, adapted from the Diploma de Español como Lengua Extranjera (DELE) (Embajada de España, Washington D.C.). The test consisted of a cloze passage and a multiple choice vocabulary test.

Desgraciadamente en ninguno de los dos casos se aportan los resultados a su investigación. Asimismo, se concede siempre menor importancia a esta prueba que a la nivelación administrativa. De hecho, es llamativo el segundo caso, puesto que ni siquiera se indica cuáles de los exámenes correspondientes a los DELE se han utilizado. Por último, sorprende el hecho de que se elige solo una parte de los exámenes y que en ambos casos está diseñada para reconocer el dominio de contenidos gramaticales, no tanto para dar información completa acerca del nivel al que pertenecen los aprendices de ELE.

Frente a este estado de cosas, debemos subrayar que es decisivo considerar el grado de dominio de ELE por parte de los individuos analizados en los estudios de adquisición. Si asumimos como cierto que en el proceso el aprendiz pasa por sucesivos estados de IL, no tiene sentido mezclar a individuos correspondientes a los diferentes estadios en el análisis o no tener en cuenta la IL en la que se encuentran en el momento en el que se lleva a cabo la investigación. Al mismo tiempo, el estudio por niveles nos va a permitir estratificar la muestra de estudio. Desde esa división sí podremos establecer y emitir juicios consistentes en relación con el proceso mismo de adquisición de ELE.

Evidentemente nuestra propuesta va por la línea de utilizar exámenes de acreditación de grado de dominio, entendidos desde los diferentes niveles que se reconocen. Hoy por hoy y para el caso del español solo los DELE garantizan un nivel de fiabilidad mínimo para la investigación, a la vez que están estructurados de modo que facilitan ese estudio estratificado.

Pero además los resultados obtenidos por los individuos analizados en las pruebas para la obtención de los DELE han de ser analizados rigurosamente con el objeto de garantizar su pertenencia a un mismo grupo. Habrá que considerar no solamente si los aprendices han obtenido alguno de los DELE, sino también habrá que considerar la 
calificación obtenida. Solo calificaciones básicamente iguales nos garantizarán que los aprendices pertenecen efectivamente a un mismo nivel de IL. El sistema de calificación de los exámenes para la obtención de los DELE prevé que la puntuación máxima que se puede obtener sea de 100 puntos, distribuidos en tres bloques ${ }^{22}$. Para obtener cualquiera de los diplomas se ha de alcanzar al menos un $70 \%$ de acierto en cada uno de los tres grupos que componen la calificación. Solo en ese caso se suman las tres calificaciones y se obtiene el apto global. Interpretaremos como del mismo nivel a aquellos estudiantes que hayan sido considerados aptos y que tengan un grado de acierto total entre el 70 y el $80 \%$ en la prueba de Expresión Escrita, que servirá de base para el corpus.

En ese sentido, queremos terminar citando el trabajo de Sánchez Iglesias (2003, p.61), como ejemplo de manera de hacer las cosas en este campo. Parte de la prueba de expresión escrita del examen para la obtención del Diploma de Español Nivel B2, antes denominado Diploma Básico de Español:

A la hora de escoger los materiales que integrarían nuestro corpus, nos inclinamos por la utilización del Diploma Básico de Español (DBE) por dos razones fundamentales. En primer lugar, porque proporciona una gran cantidad de material escrito, ya que para la evaluación de esta destreza el examen contempla la elaboración de dos textos, diferentes además desde el punto de vista de los géneros textuales. En segundo lugar, porque al ser exámenes se puede establecer rápida y fácilmente el nivel concreto de adquisición de los sujetos. Por último, seguimos un criterio de viabilidad, en tanto que este material resultaba muy accesible.

Para garantizar que todos los informantes tienen el mismo nivel, escoge solo aquellos que obtuvieron la calificación de APTO y que además lograron entre 10,5 y 12 puntos en la sección de expresión escrita; esto es, entre un 70 y un $80 \%$ de acierto. Utiliza la prueba de gramática y vocabulario como mecanismo de control para garantizar la homogeneidad de la muestra ${ }^{23}$ :

\section{Los datos y el tipo de prueba usado para conseguirlos}

Ellis (1994) distingue tres tipos de datos aportados por los aprendices y que pueden usarse en la investigación sobre ASL según la naturaleza de los mismos A ellas subyacen tres percepciones diferentes: el uso de la lengua nos informa acerca de la actuación de los aprendices, los juicios lo hacen en relación con su competencia y

\footnotetext{
${ }^{22}$ La información completa puede consultarse en la Descripción general del sistema de evaluación DELE publicado por el I. Cervantes en 2006: http://diplomas.cervantes.es/general/folletos.jsp.

${ }^{23}$ El sistema de corrección garantiza la fiabilidad de los resultados: Citamos literalmente la Descripción general del sistema de evaluación DELE publicado por el I. Cervantes en 2006: las composiciones de las pruebas de expresión escrita son calificadas por dos evaluadores que puntúan los trabajos en una escala de 0 a 15 . La nota final se calculará a partir de la nota media de ambas calificaciones. En caso de que entre ambas calificaciones se obtuviese una diferencia de más de tres puntos, el ejercicio se califica una tercera vez.
} 
los autoinformes nos facilitan datos sobre la conciencia de aprendizaje. Nos fijaremos exclusivamente en los dos primeros tipos de datos ${ }^{24}$.

Veamos qué tipo de pruebas han aplicado los investigadores de la adquisición del sistema verbal español ${ }^{25}$ :

\begin{tabular}{|c|c|}
\hline $\begin{array}{l}\text { Ti Chau } \\
(1975)\end{array}$ & Ejercicio de selección múltiple con 33 items \\
\hline $\begin{array}{l}\text { Barnwell } \\
(1987)\end{array}$ & Texto con 17 huecos (se indica el infinitivo) ${ }^{26}$. \\
\hline Stokes (1988) & $\begin{array}{l}\text { En cada bloque se escucha el principio de } 20 \text { frases. El estudiante la } \\
\text { continúa como quiere. Primer bloque: estudios universitarios. Segundo: } \\
\text { viajes y recreo, Todo se formula en presente para no complicar con otra } \\
\text { variable. }\end{array}$ \\
\hline Ramsey (1990) & $\begin{array}{l}\text { Historieta de } 10 \text { viñetas acompañadas con textos con huecos para } \\
\text { completar. La primera no tiene blancos Los huecos coinciden con los } \\
\text { verbos. El número de blancos aumenta conforme avanza la historia. No } \\
\text { indica si aporta el infinitivo. }\end{array}$ \\
\hline Hasbún (1995) & $\begin{array}{l}\text { Vídeo de } 8 \text { minutos sin voz tomado de la película de Tiempos Modernos. } \\
\text { Lo pasa } 2 \text { veces. Los estudiantes deben relatarlo en pasado. }\end{array}$ \\
\hline $\begin{array}{l}\text { Collentine } \\
(1995)\end{array}$ & $\begin{array}{l}\text { Conversación de } 10 \text { minutos donde les lleva a hacer narraciones y } \\
\text { descripciones. A continuación formula una serie de preguntas que intentan } \\
\text { forzar el uso del subjuntivo en oraciones subordinadas sustantivas. } \\
\text { Usa } 44 \text { viñetas. Cada una incluye una conversación con dos bocadillos. Se } \\
\text { hace una pregunta referida a la conversación que han leído en el dibujo y } \\
\text { que eventualmente fuerza el uso del subjuntivo }{ }^{27} \text {. }\end{array}$ \\
\hline Lafford (1996) & $\begin{array}{l}\text { Vídeo mudo de } 10 \text { minutos tomado de la película Aprendiz de Brujo. Los } \\
\text { estudiantes tienen que contar la historia en pasado. }\end{array}$ \\
\hline
\end{tabular}

${ }^{24}$ Poco podemos aportar a las afirmaciones de Ellis referidas a los autoinformes, y su importancia para analizar los factores afectivos y cognitivos del proceso de adquisición, con todas las limitaciones que este tipo de datos exhiben y que muy acertadamente expone en su trabajo. En Bustos Gisbert y Sánchez Iglesias (2006) utilizamos precisamente los autoinformes para estudiar la conciencia de fosilización de errores en los buenos aprendices de ELE.

${ }^{25}$ A la izquierda aparece la referencia al trabajo del investigación. En la parte derecha indicamos si se han usado muestras de lengua de los aprendices o si aparecen las tareas usadas vinculadas a juicios metalingüísticos.

${ }^{26} 9$ indefinidos, 7 imperfectos y 1 donde ambos son correctos. 4 verbos son pronominales.

${ }^{27}$ Hay 50 preguntas. 20 son distractores y 30 provocan el uso de subordinadas sustantivas: de ellas 15 exigen indicativo y 15 exigen subjuntivo. Se graban las respuestas. 


\begin{tabular}{|c|c|}
\hline Güell (1998) & $\begin{array}{l}\text { Narración "no guiada" de una historia: acota las opciones de continuación } \\
\text { con una historia gráfica tomada de un método de enseñanza de ELE. } \\
\text { Tienen que usar las expresiones El otro día y Al día siguiente. Los } \\
\text { estudiantes tienen que escribirla. } \\
\text { Texto de huecos (se indica el infinitivo) en una narración de pasado. Se les } \\
\text { pide que usen la forma verbal adecuada } \\
\text { Prueba de selección múltiple. Es el mismo texto que en la prueba anterior. } \\
\text { Se sustituyen los indefinidos por las formas de imperfecto e indefinido. } \\
\text { Prueba de selección múltiple: ahora son oraciones con el imperfecto y el } \\
\text { indefinido para que elijan uno de ellos }{ }^{29} \text {. }\end{array}$ \\
\hline $\begin{array}{l}\text { Salaberry } \\
\text { (1999) }\end{array}$ & $\begin{array}{l}2 \text { fragmentos de la película Tiempos } \text { Modernos }^{30} \text {. Se hace una } \\
\text { dramatización compleja donde un estudiante cuenta a otro el fragmento } \\
\text { como si fuera un caso policiaco }^{31} \text {. }\end{array}$ \\
\hline $\begin{array}{l}\text { Montrul y } \\
\text { Slabakova } \\
(1999)\end{array}$ & $\begin{array}{l}\text { Test de selección múltiple. Se elige entre dos formas (imperfecto e } \\
\text { indefinido) })^{32} \text {. } \\
\text { Tarea reflexiva en la que se juzga la lógica de las conjunciones (Y o } \\
\text { PERO) en oraciones. Tal lógica depende de que aparezca imperfecto o } \\
\text { indefinido }^{33} \text {. }\end{array}$ \\
\hline $\begin{array}{l}\text { Cadierno } \\
(2000)\end{array}$ & $\begin{array}{l}\text { Entrevista. } \\
\text { Composición. } \\
\text { Dos textos de huecos (se indica el infinitivo). Se han de rellenar con la } \\
\text { forma verbal del pasado correcta (imperfecto o indefinido). Además, los } \\
\text { estudiantes deben dar una explicación en hoja aparte de por qué han }\end{array}$ \\
\hline
\end{tabular}

${ }^{28}$ Describe la naturaleza semántica de los verbos pero no se exige que los verbos reflejen un equilibrio absoluto en términos de su contenido léxico inherente.

${ }^{29}$ Esta prueba debe servir para comprobar si elijen realmente por criterios aspectuales. Entiende que las dos anteriores no lo dejan claro así que elimina el contexto temporal explícito.

${ }^{30}$ Ya usados en el European Science Foundation Project para francés, inglés y español como L2.

31 Se entrevista por pares de estudiantes del mismo nivel. Uno es un testigo de un acontecimiento y se lo cuenta a un detective de la policía. Después el detective se lo cuenta al jefe de detectives (que es un nativo o cuasi nativo). El estudio tiene carácter longitudinal: se recogen los datos en dos ocasiones durante el proceso de instrucción para comprobar el grado de desarrollo de su aprendizaje. Además se ejerce presión de tiempo y de urgencia comunicativa.

${ }^{32}$ Es un texto narrativo completo con 30 ítems que representan los cuatro grupos semánticos de verbos. 15 se contestan con imperfecto y 15 con indefinido. Solo vale uno en cada ítem.

${ }^{33}$ Se evalúa desde -2 (ilógico) hasta 2 (lógico), incluyendo valoraciones intermedias. Hay 56 frases, 28 lógicas y 28 ilógicas. Se distribuyen de la siguiente manera: 14 logros, 14 realizaciones, 14 estados. En cada una hay 7 de imperfecto y 7 de indefinido. 14 frases son distractores que usan cualquier verbo, no solo pasados.

${ }^{34}$ Con las dos partes quiere comparar la producción efectiva con el conocimiento metalingüístico. 


\begin{tabular}{|c|c|}
\hline & elegido una $\mathrm{u}$ otra forma ${ }^{34}$. \\
\hline $\begin{array}{l}\text { Díaz y Bekiou } \\
(2006)\end{array}$ & $\begin{array}{l}\text { Los estudiantes tienen que contar una historieta muda en tercera persona. } \\
\text { Se consignan las pausas, las repeticiones y las reparaciones en el discurso } \\
\text { oral. }\end{array}$ \\
\hline $\begin{array}{l}\text { Gudmestad } \\
(2006)\end{array}$ & Ejercicio de selección múltiple con dos opciones ${ }^{35}$. \\
\hline $\begin{array}{l}\text { Di Franco } \\
(2007)\end{array}$ & $\begin{array}{l}\text { Se analizan } 7 \text { redacciones de cada aprendiz escritas a lo largo de un curso } \\
\text { académico. No proporciona datos sobre la naturaleza de las redacciones. }\end{array}$ \\
\hline $\begin{array}{l}\text { Sánchez } \\
\text { Castro (2008) }\end{array}$ & $\begin{array}{l}\text { Redacción de } 200 \text { palabras, dirigida al uso de imperfecto y perfecto simple. } \\
\text { Básicamente narrativa. }\end{array}$ \\
\hline $\begin{array}{l}\text { Cuza Blanco } \\
(2008)\end{array}$ & $\begin{array}{l}\text { Expresión oral controlada: se pide que cuenten en pasado el cuento de } \\
\text { Caperucita Roja y les dan } 6 \text { imágenes sin palabras que les sirven de } \\
\text { apoyo }^{36} \text {. } \\
\text { Juicio de verdad: Se evalúa la verdad o mentira de los enunciados sobre la } \\
\text { base del uso de imperfecto e indefinido }{ }^{37} \text {. } \\
\text { Juicio de aceptabilidad: Se evalúa la aceptabilidad del uso de imperfecto o } \\
\text { indefinido en frases donde el opuesto es la forma preferida }{ }^{38} \text {. }\end{array}$ \\
\hline Mao, P. (2009) & $\begin{array}{l}\text { Redacción. } \\
\text { Texto de huecos (se indica el infinitivo) para completar con } \\
\text { imperfecto/indefinido }\end{array}$ \\
\hline
\end{tabular}

Cuadro 7. Pruebas aplicadas al estudio de la adquisición del sistema verbal

Podemos resumir los datos de la siguiente manera desde la naturaleza de la prueba administrada:

${ }^{35}$ Una historia en inglés que ha sido dividida en 35 fragmentos. En cada uno se dan dos frases en español para elegir una. Solo se diferencian en el modo: 20 recogen contextos donde se usa el subjuntivo. Las opciones están codificadas con 4 variables: verbo irregular, valor de futuro, valor de deseo y valor de emoción. 15 exigen indicativo y se usan como distractores Evalúa la prueba como muy cómoda para analizar los datos: da información oral y contextual de forma simultánea. Pero reconoce que los datos no son generalizables y no nos informan acerca del uso real.

${ }^{36}$ Se obtuvieron 60 respuestas de 168 a 587 palabras en el grupo de control, de 100 a 400 en el grupo de aprendices, y de 200 a 300 entre los inmigrantes.

37 Se presenta un pasaje breve en inglés seguido de una construcción en español. Sólo necesita elegir si lo que se dice es verdad o no de acuerdo con lo que se plantea en el pasaje. Se aplica de forma oral mediante un ordenador donde se graban los resultados para su posterior valoración. No consta el número de ítems.

${ }^{38}$ Se evalúa la aceptabilidad de 1 a 5 . Se hace de manera escrita con 40 frases: se lee el contexto, se lee la frase donde se usa imperfecto o indefinido y se indica el nivel de aceptabilidad de la frase alternativa.

${ }^{39}$ Verbos clasificados en 4 grupos desde un punto de vista semántico. 


\begin{tabular}{llll}
\hline & Uso de lengua & $\begin{array}{l}\text { Juicio } \\
\text { Metalingüístico }\end{array}$ & Uso y Juicio \\
\hline Tipo de prueba & 8 & 5 & 4 \\
\hline \multicolumn{4}{c}{ Cuadro 8a. Tipos de ejercicios utilizados } \\
\end{tabular}

Dentro de las dos opciones existentes, se han propuesto los siguientes ejercicios:

\begin{tabular}{lll}
\hline Uso de lengua & Redacción & 3 \\
\cline { 2 - 3 } & Redacción y entrevista & 1 \\
& Dramatización & 1 \\
& Reformulación oral en pasado & 5 \\
& Conversación guiada & 1 \\
& Continuación de enunciados & 2 \\
Juicio & Texto de huecos con indicación del infinitivo & 5 \\
Metalingüístico & Texto de huecos y justificación de la elección & 1 \\
& realizada & 2 \\
& Selección múltiple & 1 \\
& Reflexión sobre la lógica de enunciados & 1 \\
\hline & Juicio de verdad de enunciados & 1 \\
\hline
\end{tabular}

Cuadro 8b. Tipos de ejercicios utilizados

Los procedimientos de obtención de datos utilizados por los investigadores estudiados y vinculados a juicios son básicamente de dos tipos. Una opción es la pura reflexión metalingüística acerca de determinados enunciados: puede tomar la forma de justificar más o menos teóricamente el porqué de la selección de una forma verbal o bien puede ser la valoración sobre la lógica, la verdad o el grado de aceptabilidad de determinados enunciados según la forma verbal usada. En todos los casos se realizan estas pruebas para establecer evaluaciones sobre el conocimiento de las diferencias aspectuales derivadas del uso de las formas de imperfecto o indefinido.

La segunda posibilidad ya la citamos antes cuando analizábamos el uso de la lengua y tiene que ver con la utilización de ejercicios focalizados en la forma gramatical; asumen dos grandes formas: o bien se plantean ejercicios de huecos con indicación del infinitivo o bien se dan las formas verbales ya conjugadas para que el aprendiz elija la que le parezca más conveniente, en la forma habitual de una actividad de selección múltiple. El planteamiento es siempre una distinción binaria, ya sea entre imperfecto o indefinido, o entre indicativo y subjuntivo.

En relación con los juicios metalingüísticos sustentados en la pura reflexión sobre el grado de validez de los enunciados, hay que decir que nos dan datos sobre el conocimiento competencial, pero se trataría como mucho de un dominio teórico, reflexivo y por ello metaconceptual que no garantiza que el aprendiz use tales contenidos. En otras palabras, dudo que se pueda hablar de adquisición sin detección 
de uso efectivo ${ }^{40}$. A eso se añaden las críticas que el propio Ellis, siguiendo a Birdsong (1989), reconoce y que tienen que ver con cuánto de fiables son los juicios de gramaticalidad de los hablantes nativos que normalmente funcionan como grupos de control.

En segundo término, los juicios basados en procedimientos de huecos, estén más o menos explicitadas las opciones, plantean problemas vinculados a la interpretación de los resultados y no podemos aceptar como argumento que efectivamente faciliten la investigación sobre propiedades lingüísticas que de otro modo (y estamos citando casi literalmente a Ellis) serían de difícil estudio. Los problemas son de cuadruple naturaleza: en primer término, tienen que ver con que el grado de acierto o error en la resolución de las actividades no garantiza un grado de acierto o error equivalente en el uso efectivo; por otro lado, en cualquier ejercicio de esta naturaleza será difícil evaluar el efecto de la respuesta aleatoria, sea esta acertada o incorrecta; en tercer lugar, en los ejercicios basados en textos con huecos se ha observado como un uso incorrecto puede generar una cadena de usos erróneos sin que ellos tenga que ver con otra cosa que no sea con la naturaleza discursiva del ejercicio; por último, la propia construcción de este tipo de ejercicios demuestra la dificultad de evitar las posibles dobles respuestas correctas, salvo en los casos en los que se opta por procedimientos de selección múltiple donde las opciones creadas en el fondo no cumplen con su función distractora.

Por todo ello, los juicios metalingüísticos son los que más recelos nos despiertan: no es tan evidente el establecimiento de una correlación automática entre la adecuación del juicio y el grado de adquisición del concepto y quedan demasiadas dudas referidas a la propia interpretación de los resultados. El juicio puede usarse pero no como fuente exclusiva de datos en tanto que no exhibe el nivel de la actuación de los aprendices; es decir, se han de validar los resultados obtenidos con su aplicación con datos extraídos del uso real de la lengua, sea este más o menos dirigido.

Por lo que afecta al uso de la lengua, no coincidimos plenamente con las distinciones que hace Ellis en lo relativo a los tipos de uso de la lengua, especialmente en lo que afecta al uso provocado de naturaleza clínica y experimental. Efectivamente, el primero de ellos, el uso efectivo, nos servirá para detectar el estatus de la actuación y también de la competencia de los aprendices. El dominio del sistema subyace al uso. Sin embargo, no podemos suscribir los tipos de prueba que asocia al uso guiado. Este propone un grado de libertad restringido; sí, pero libre al fin y al cabo. Permite al aprendiz optar entre opciones expresivas. Le posibilita incluso obviar estratégicamente algunas opciones. Y todo esto lo encontramos en alternativas como la redacción o la entrevista oral más o menos guiadas y con independencia del grado de control que se quiera establecer en una u otra. Por poner un ejemplo en el ámbito

${ }^{40}$ Es difícil medir el grado de adquisición en pruebas que no sean de aplicación de la competencia comunicativa entendida en términos globales. Todo lo demás supone una manipulación que está bien para clase en tanto que estrategia docente, pero que no refleja grado de adquisición real. 
de la expresión oral, una dramatización puede ser tan guiada o más que un ejercicio de retelling. Y todo esto, a su vez, nada tienen que ver con ejercicios de lectura en voz alta, de traducción, de reconocimiento de estructuras o de utilización de ítems discretos, mucho más cerca de los juicios metalingüísticos que del uso.

\section{1 Lengua oral o lengua escrita}

Las investigaciones tienden a utilizar indistintamente muestras de lengua oral o escrita y solo en algunos casos se aplican actividades que obligan a los aprendices a realizar tareas en ambas modalidades. De los 17 trabajos de los que disponemos de información completa referida al tipo de prueba realizada, 6 parten de lengua oral, 8 lo hacen de lengua escrita y 3 usan muestras de una y de otra.

Para el caso del estudio de la adquisición de la temporalidad entendemos que resulta más recomendable la utilización de muestras de lengua escrita. Existen al menos cuatro argumentos a nuestro favor.

En primer lugar, por la propia naturaleza del texto conversacional. En ese sentido, son aclaratorias las conclusiones de Briz $(1998)^{41}$, quien defiende que carecemos de un estudio del paradigma verbal en la conversación coloquial, muy especialmente en lo que afecta a gramaticalizaciones y especializaciones pragmáticas. Briz destaca solo algunas variaciones esenciales de la temporalidad en el coloquio, como son los usos del presente en la expresión de acciones pasadas ${ }^{42}$; la transformación del futuro, que deja de establecer diferencias temporales para pasar a expresar valores modales; la ampliación de los usos del imperfecto de indicativo para la expresión modalizada (de deseo, cortesía, afirmación atenuada...); y las frecuentes neutralizaciones en el modo verbal.

A esto ha de añadirse la propia especificidad sintáctica del discurso oral y que afecta de manera evidente a la construcción de la temporalidad. Cabe destacar algunas de estas características, como el uso de sintaxis concatenada ${ }^{43}$, merced a la cual se prefiere una construcción encadenada frente a la incrustada del registro formal escrito; la parcelación y la simplificación sintáctica del discurso conversacional; la organización pragmática y no sintáctica del discurso, donde el orden de las palabras se realiza en virtud de los objetivos informativos de las unidades; o las dependencias sintácticas que derivan de la propia interacción conversacional.

En segundo lugar, porque la lengua conversacional se basa en cooperación y en estrategia ${ }^{44}$. Cooperación con el tema y con el interlocutor. Estrategia en la medida en que comunicar no es solo codificar y descodificar, sino también y sobre todo mostrar e inferir. La interacción coloquial genera especificidades expresivas derivadas de la negociación de los turnos conversacionales. Así, por ejemplo la redundancia funciona como recurso para dar continuidad al discurso, ya sea para lograr o para retener el turno. Del mismo modo, la construcción sintáctica se hace interactiva, y los

\footnotetext{
${ }^{41}$ Que a su vez se inspira en Bustos Tovar (1995) y Narbona (1989).

42 En lo que habitualmente denominamos "presente resumen".

${ }^{43}$ Véase Narbona (1989).

${ }^{44}$ Sigo en lo esencial a Briz (1998).
} 
enunciados de uno influyen en los del otro. Este hecho, a la hora de valorar el grado de adquisición del sistema verbal en aprendices de L2 puede incluir variables no previstas, como es el caso del nivel de influencia de las formas de expresión usadas por un interlocutor en el otro: el interlocutor puede dar la forma correcta sin que ello presuponga dominio; y al revés, puede contaminar e inducir a un error que en otra situación comunicativa no tendría por qué haberse producido. Más cuando ambos son aprendices de L2.

Se podrá argüir entonces que podemos optar por una expresión oral monologal, como hacen buena parte de los trabajos estudiados ${ }^{45}$. El problema de la construcción monologal es que no se trata de una modalidad de expresión a la que los aprendices de L2 están acostumbrados. De hecho, tampoco lo es en el caso de la L1. Por ello, parece contradictorio verificar el grado de adquisición de un contenido lingüístico especializado sobre la base de una modalidad expresiva que habitualmente ni siquiera dominamos en nuestra primera lengua.

En tercer lugar ha de tenerse en cuenta que la descripción normativa del estándar, que es la que se utiliza habitualmente para verificar el grado de adquisición, se hace desde el registro formal escrito y especializado. Efectivamente, los procesos de estandarización establecen relaciones inmediatas con los diferentes registros que se detectan en el uso. De manera general, las variedades estándar se identifican con los registros formales.

Por último, disponemos no solo de una descripción gramatical estandarizada del sistema verbal en nuestra lengua, sino también de datos fiables en lo que tiene que ver con su uso en la variable escrita de la lengua y con referencia a los cuatro grandes tipos de secuencias textuales: narrativas, descriptivas, expositivas e instructivas. En Bustos Gisbert (2006) hemos realizado con una descripción del uso del sistema verbal del español en un corpus de aproximadamente 228 secuencias textuales correspondientes a 35 textos distintos y que incluyen aproximadamente 70.000 palabras ${ }^{46}$.

\section{Conclusión}

Los estudios de ASL deben sustentarse en una metodología consistente en lo que toca a la muestra de aprendices seleccionada para realizarla,. Y lo debe ser en dos

${ }^{45}$ Ya sean en forma de resumen o de reformulación oral.

${ }^{46}$ En el estudio se incluye en primer lugar una serie de consideraciones generales que tiene que ver con un análisis global de la temporalidad en cada grupo de secuencias; aporta datos en relación con los tiempos verbales prototípicos y con los de uso nulo o testimonial; también se han establecido comparaciones del uso en una secuencia frente a los resultados globales. En segundo término, se ha estudiando la influencia de la extensión secuencial en la configuración del sistema verbal. Finalmente se ha estudiando el rendimiento de los diferentes tiempos verbales; para ello se ha estudiado el uso de los tiempos prototípicos y se ha prestado especial atención al valor estilístico y a la función textual de cada tiempo verbal en los conjuntos tipológicos. 
grandes direcciones: en lo que afecta al tamaño mismo de la misma y en lo que toca al nivel de los informantes seleccionados.

Las elecciones muestrales en la investigación de ASL se realizan sin consistencia estadística. Se tiende a elegir a los aprendices que se tiene más a mano. En consecuencia, son grupos cuya representatividad no se puede garantizar ni en términos cuantitativos ni cualitativos. Asimismo, la consideración de la L1 de los aprendices no siempre es una variable tenida en cuenta a la hora de establecer diferencias en la selección de informantes. Sin embargo, parece que la lengua materna puede ser una herramienta perfectamente adecuada para la estratificación de la muestra, toda vez que la mayoría de los estudios de adquisición asume la importancia de los procesos de transferencia. En tercer lugar, con mucha frecuencia se mezclan aprendices correspondientes a diferentes estados de IL. El nivel de dominio de ELE es la segunda variable clave en los procesos de estratificación $\mathrm{y}$, por ello, de organización de los estudios de adquisición. De hecho, así lo demuestran los avances alcanzados en el ámbito de los trabajos dedicados al análisis de los distintos niveles de IL de los aprendices. Por todo ello, los resultados obtenidos hasta ahora han de ser relativizados en la medida en que difícilmente resultan generalizables. Son aportaciones valiosas que nos indican la dirección por la que se pueden mover nuestras intuiciones, pero no pueden ser interpretados como definitivos

Por su parte, en lo que toca la al nivel de los aprendices estudiados, buena parte de los estudios realizados nivela a los estudiantes a partir de argumentos administrativos. Pertenecer a un mismo grupo de estudiantes es uno de los parámetros más utilizados. Pero esta variable no nos garantiza que tengan un grado de conocimiento similar. De hecho, algunas pruebas de los propios investigadores así lo indican. Lo mismo ocurre cuando complementamos el dato administrativo con el número de horas de clase que han recibido, que es otro de los que habitualmente aparecen. En pocas ocasiones se realizan pruebas para garantizar el nivel, o se usan procedimientos de nivelación poco fiables basados. Con frecuencia se hacen presuposiciones no muy consistentes acerca de la instrucción previa que han recibido las personas que componen la muestra. Además, no importa solo el modelo instructivo sino también los contenidos que han configurado el input. No se utiliza de manera explícita el Marco de Referencia para establecer el nivel de dominio de ELE por parte de los aprendices estudiados

Ante estas circunstancias, proponemos la realización de estudios de adquisición de ELE sobre la base de muestras construidas de forma fiable sobre la base del universo de aprendices que nos proporcionan las pruebas para la obtención de los DELE. Defendemos, además, que la muestra se estratifique sobre la base de dos grandes variables, como son la lengua materna y el nivel de los aprendices.

El número total de candidatos que se presentaron a las pruebas de los DELE entre 1989 y 2006 es de 286.000 , lo cual configura un universo de partida realmente amplio. No obstante, necesitamos depurar estos datos para que reflejen un universo de estudio fiable. Para ello, nos detendremos en los datos correspondientes a los candidatos presentados a las pruebas para la obtención del Diploma Básico de Español, equivalente al actual Diploma de Español Nivel B2 (Intermedio) entre 1992 y 1999, a los que hemos tenido acceso y que nos servirán como ejemplo para valorar 
la consistencia del corpus propuesto: el número total de candidatos presentados a las pruebas es de 55.792. De ellos, se consideraron aptos 40.491; esto es, un 72,5\% de los candidatos que se presentaron. A su vez, de ellos obtuvieron una calificación entre 10,5 y 12 en la prueba correspondiente a Expresión Escrita (entre un 70 y un $80 \%$ de acierto) 10.400 candidatos, que son un $18,6 \%$ del total de los presentados y un $25,68 \%$ de los que fueron considerados aptos.

Nuestro punto de partida hipotético sería de más de 10.000 informantes; considerando el crecimiento de los DELE en los últimos 10 años, ese corpus se multiplicaría por dos o por tres. Las cifras se pueden valorar conforme al gráfico siguiente $^{47}$ :

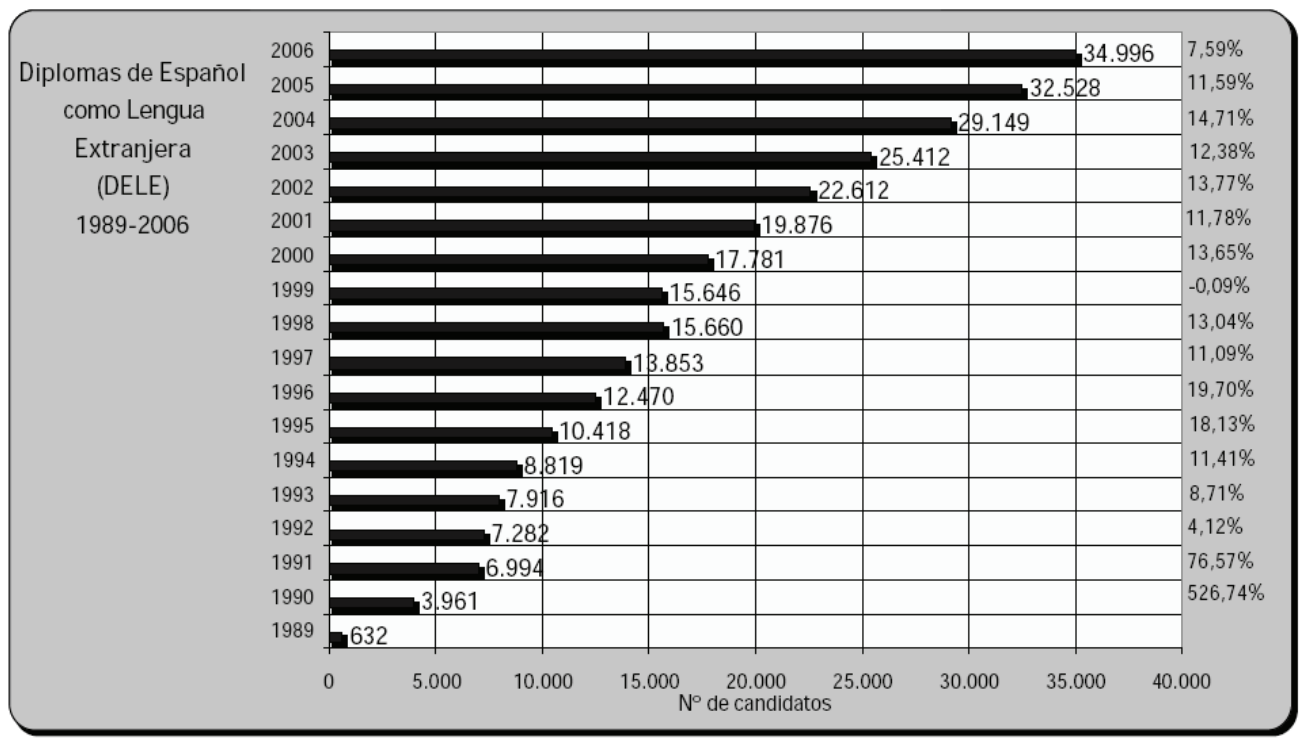

Cuadro 9. Candidatos que han realizado los exámenes para obtener los Dele

En lo que afecta a su lengua materna, no disponemos de datos absolutamente exactos, y sería necesario revisar las pruebas escritas de los aprendices. No obstante, en la actualidad, los candidatos a los DELE indican explícitamente su lengua materna, por lo que sería fácil hacer la distribución correspondiente. En cualquier caso, sobre la base de trabajo podría hacerse un análisis basado en el lugar de nacimiento indicado por las personas que hicieron las pruebas y en la ciudad en la que éstas tuvieron lugar.

Si nos fijamos en el tipo de prueba, observamos que se utilizan indistintamente ejercicios donde el aprendiz se expresa en lengua hablada o en lengua escrita, sin que

${ }^{47}$ Tomado de la Descripción general del sistema de evaluación DELE publicado por el I. Cervantes en 2006. El documento se puede consultar en http://diplomas.cervantes.es/general/ folletos.jsp 
se justifique casi nunca la razón por la que se escoge uno u otra. Además, se usan pruebas de uso libre y de juicio metalingüístico al $50 \%$ ya sea de forma exclusiva o combinada. Se enmascaran en ocasiones juicios metalingüísticos como si fueran pruebas de uso libre. Se alegan normalmente dificultades de realización a la hora de justificar por qué se renuncia a las pruebas de uso libre. Finalmente, no se justifica el modelo de interpretación de los datos cuando se aplican pruebas destinadas a evaluar la adquisición del sistema verbal. Bien puede suceder que no se sepa si el mal uso de un verbo se debe a un desconocimiento de la forma a que no se dominan las posibles funciones de cada tiempo. Por ejemplo, no sabemos si los aprendices confunden la forma del imperfecto y del indefinido o si no saben cuándo se usa uno y otro. Lo mismo ocurriría en el caso del modo: no podemos decidir si no saben el subjuntivo como forma o desconocen cuándo se usa ${ }^{48}$.

Defendemos el uso libre de la lengua como muestra sobre la que efectuar la investigación. Entre otras cosas, porque el texto nos va a proporcionar mucha más información sobre la adquisición en tanto que nos va a permitir cotejar aciertos y errores de uso contextualizados. Y el contexto nos va a permitir dilucidar la naturaleza de los errores detectados. En una actividad donde el aprendiz informante se limita a escribir una forma verbal no podremos nunca realizar otro tipo de interpretaciones. Proponemos para el estudio de la adquisición del sistema verbal español el uso de las pruebas de expresión escrita. En este caso las correspondientes a los exámenes para la obtención de los DELE. Son pruebas guiadas en las que sin embargo, el grado de libertad expresiva es razonablemente alto o muy alto ${ }^{49}$.

Entendemos que los exámenes para la obtención de los DELE nos van a proporcionar en cada nivel de IL una variedad de textos suficiente como para hacer la muestra perfectamente representativa. Por otra parte, los resultados en el resto de las pruebas, y específicamente la prueba destinada al dominio formal del español, podrían perfectamente servir como prueba de control. Recordemos que los ítems de dicha prueba están construidos a partir de un listado de estructuras formales prediseñado. Bastaría con escoger aquellas que afectan al dominio del sistema verbal para establecer las comparaciones oportunas. Pero eso ya es materia para otro trabajo.

\section{OBRAS CITADAS}

ALEXOPULU, Angélica: Análisis de errores en la interlengua de aprendientes griegos de español, Madrid, Ediciones del Orto, 2006.

ARKIN, Herbert: Tables for statisticians, New York, Barnes \& Noble, 1968.

BACHMAN, Lyle. F. y Andrew D. COHEN (eds.): Interfaces between second language acquisition and language testing research, Cambridge, Cambridge University Press, 1998.

${ }^{48}$ Esta reflexión aparece ampliamente desarrollada en Montrul y Slabakova (2002).

49 Véase la Descripción general del sistema de evaluación DELE publicado por el I. Cervantes en 2006. 
BARALO, Marta: "La adquisición del subjuntivo en el español nativo y no nativo". En En M. Villayandre (ed.): Actas del XXXV Simposio de la Sociedad Española de Lingüística, León, Universidad de León, 2006, pp. 307-318.

BARALO, Marta: "La alternancia imperfecto-indefinido en el español no nativo". En P. Cano (coord.): Actas del VI Congreso de Lingüística General, Santiago de Compostela, 3-7 de mayo de 2004. Madrid, Arco, 2007, pp. 339-346.

BARNWELL, Davis: Syntactic and morphological errors of English speakers on the Spanish past tenses, Columbia University, Research report, 1987.

BELlido, Óscar: La voz pasiva perifrástica en las lenguas española e inglesa, Universidad de Salamanca, Tesis doctoral, 1989.

BELTRÁN, Miguel: "Cinco vías de acceso a la realidad social". En M. García Ferrando, J. Ibáñez y F. Alvira (comps.): El análisis de la realidad social. Métodos y técnicas de investigación, Madrid, Alianza, 2003, pp.15-55.

BERDUGO, Óscar: "Reflexiones sobre el español como recurso económico". Contrastes, 39 (2005), pp. 53-58.

BertinetTo, Pier Marco y Denis Delfitto: "L' Espressione della ProgressivitàContinuità: Un Confronto Tripolare Iitaliano, Inglese e Spagnolo" En P. Benincà et al. (eds.): Italiano e Dialetto Nel Tempo: Studi di Grammatica Offerti a Giulio Lepschy, Rome, Bulzoni, 1996, pp. 45-66.

BRILL, Jana. A.: Past times in French: a study of the passé simple - passé composé distribution, with reference to Spanish and Italian, Ann Arbor, University Microfilms International, 1984.

BRINDLEY, Geoff: "Describing language development? Rating scales and SLA". En L. F. Bachman y A. Cohen (eds.): Interfaces between second language acquisition and language testing research, Cambridge, Cambridge University Press, 1998, pp. 112-140.

BRIZ, Antonio: El español coloquial en la conversación. Esbozo de pragmagramática, Barcelona, Ariel, 1998.

BROWN, Gillian. y George Yule: Teaching the spoken language, Cambridge, Cambridge University Press, 1983.

BugedA, José: Manual de técnicas de investigación social, Madrid, Instituto de Estudios Políticos, 1974.

BURR, Elisabeth: "Il sistema verbale italiano e spagnolo. Indagine sulla lingua dei quotidiani”. En F. Foresti, E. Rizzi y P. Benedini, P. (eds.): L'italiano fra le lingue romanze. Atti del XX Congresso Internazionale di Studi, Bologna 25-27 settembre, Roma, Bulzoni, 1989, pp. 253-276.

Bustos GisBerT, José Manuel y J. SÁNCHEZ IGLESIAS (coords.): La fosilización en la adquisición de segundas lenguas: el buen aprendiz, Salamanca, Luso Española de Ediciones, 2006.

Bustos Gisbert, José Manuel: "Secuencia textual y temporalidad verbal". En P. Elena (coord.): Gramática y Traducción, Salamanca, Ediciones Universidad de Salamanca, 2006, pp. 11-90. 
Bustos Tovar, José Jesús: "De la oralidad a la escritura", en L. Cortés (ed.): El español coloquial. Actas del I Simposio sobre análisis del discurso oral, Almería, Universidad de Almería, 1995, pp. 11-28.

CADIERNO, Teresa: "The acquisition of Spanish aspectual grammatical aspect by Danish advanced language learners. Spanish Applied Linguistics, 4-1 (2000), pp. $1-53$.

CAMPS, Joaquim: "Aspectual distinctions in Spanish as a foreign language: The early stages of oral production", International Review of Applied Linguistics, 40 (2002), pp. 179-210.

CAMPS, Joaquim: "Preterit and imperfect in Spanish: The early stages of development". En R. P. Leow and C. Sanz (eds.): Spanish Applied Linguistics at the Turn of the Millennium, Somerville-MA, Cascadilla Press, 2000, pp. 1-19.

CARRERA, Miguel y José Jesús GÓMEZ ASENCIO: La economía de la enseñanza del español como lengua extranjera, Barcelona, Ariel, 2009.

CARTAGENA, Nelson. y Hans Martin GAUGER: Vergleichende Grammatiken Spanisch-Deutsch, Mannheim, Bibliographisches Institut F.A Brockhaus AG, 1989.

CARTAGENA, Nelson: "Acerca del rendimiento funcional de los paradigmas de futuro en español y en alemán.” En E. L. Traill (coord.): Scripta Philologica in Honorem Juan M. Lope Blanch Volumen II: Lingüistica española e iberoamericana, México, UNAM, 1992, pp. 65-74.

CARTAGENA, Nelson:"Das Tempussystem der spanischen Gegenwartssprache". En R. Thieroff and J. Ballweg (eds): Tense Systems in European Languages, Tübingen, Niemeyer, 1994, pp. 173-190.

CASORRÁn, Santiago: Análisis contrastivo de la modalidad verbal, Universidad de Valencia, Tesis doctoral, 1992.

CHAU, Tran-Ti: "Error Analysis, Contrastive Analysis and student perception". IRAL, 13 (1-4) (1975), pp. 119-144

COHEN, Andrew: "Strategies and processes in test taking and SLA". En L. F. Bachman, y A. Cohen (eds.) (1998): Interfaces between second language acquisition and Language testing research, Cambridge, Cambridge University Press, 1998, pp. 90-111.

COllentine, Joseph: "The development of complex syntax and mood selection abilities by intermediate level learners of Spanish". Hispania 78-1 (1995), pp. 122135.

CONSEJO DE EUROPA: Marco de referencia europeo para el aprendizaje, la enseñanza y la evaluación de lenguas. Alcalá de Henares, Instituto Cervantes, 2001.

COWPER, Elizabeth: Parameters of INFL: English Aspect and Spanish Tense, University of Toronto Syntax Project, 2003.

CuZA BLANCO, Alejandro: The L2 Acquisition and L1 Attrition of the Interpretation and Use of Aspectual Properties in Spanish among English-speaking L2 Learners and Long-term Spanish Immigrants, University of Toronto, Tesis Doctoral, 2008. 
DI FRANCO, Cinzia: "Adquisición de la selección modal indicativo/subjuntivo por estudiantes italianófonos de español: análisis de los errores más habituales". Revista Nebrija, 1-1 (2007), pp. 127-135.

DÍAZ, Lourdes y Konstantina Bekiou: "Lo que las reformulaciones y repeticiones halladas en los relatos orales de los aprendices de español L2 pueden decirnos acerca de la adquisición del aspecto verbal del español". En M. Villayandre (ed.): Actas del XXXV Simposio de la Sociedad Española de Lingüística, León, Universidad de León, 2006, pp. 400-418.

DOUGLAS, Dan: "Performance consistency in second language acquisition and language testing research: a conceptual gap". Second language research, 17-4 (2001), pp. 442-456.

DUBSKY, Josef: "Aspect du verbe et l'action verbale en français et en espagnol." Sborník Praci Filosofické Fakulty Brnełnské Univ. 10-49 (1961), pp. 157-64.

EGGS, Eckehard: "Die Vergangenheitstempora im Spanischen und Französischen". En C. Schmitt (ed.), Grammatikographie der romanischen Sprachen, Bonn, Roman Verlag, 1993, pp. 97-134.

ELLIS, Rod: The study of second language acquisition, Oxford, Oxford University Press, 1994.

ESTAPÁ, Roser. y Brian MOTT: "Algunas consideraciones sobre el futuro en inglés y en español”. BELLS, 1 (1989), pp. 71-86.

FERNÁNDEZ, Jesús: Los sistemas verbales del español y el inglés, Universidad de Salamanca, Tesis doctoral, 1990.

FERNÁNDEZ, $\mathrm{M}^{\mathrm{a}}$ Sonsoles: Interlengua y análisis de errores en el aprendizaje de español como lengua extranjera, Madrid, Edelsa, 1997.

GARCíA FERRANDO, Manuel: Socioestadística, Madrid, Alianza, 2000.

GARCÍA PAREJO, Isabel y Théophile AMBANDIANG: “Organización del componente morfológico y errores de aprendizaje: el caso de la flexión verbal en inglés y español". Didáctica (Lengua y literatura), 11 (1999), pp.55-76.

GRIFFIN, Patrick y P. NIX: Educational assessment and reporting, Sydney, Harcourt Brace Jovanovich, 1991.

GUDMESTAD, Aarnes: "L2 Variation and the Spanish Subjunctive: Linguistic Features Predicting Mood Selection". En C. A. Klee and T. L. Face (eds.): Selected Proceedings of the 7th Conference on the Acquisition of Spanish and Portuguese as First and Second Languages, Somerville-MA, Cascadilla Proceedings Project, 2006, pp. 170-184.

GÜELL, Lourdes: La adquisición del tiempo verbal en el aprendizaje de español como lengua extranjera, Universidad Autónoma de Barcelona, Tesis doctoral, 1998.

GUTIÉRREZ QUINTANA, Esther: Análisis de errores gramaticales de italianos estudiantes de español, Universidad de Alcalá de Henares, Tesis doctoral, 2003.

GUTIÉRREZ QUINTANA, Esther: Contrastes, español para brasileños, Madrid, SGEL, 2006.

GUTIÉRREZ QUINTANA, Esther: Contrastes, español para hablantes de italiano, B1B2, Madrid, SGEL, 2006. 
GuZMÁn TIRADO, Rafael y Manuela HERRADOR DEL PINO: "Dificultades en el aprendizaje del sistema verbal español para los alumnos rusos-hablantes". En A. Martínez González, J. A. de Molina Redondo y P. Pedro Barros García (eds.): II Jornadas sobre aspectos enseñanza del español como lengua extranjera, Granada, Universidad de Granada, 1993, pp. 89-94

HALLIDAY, Michael A.K: "The Users and Uses of Language". En J. A. Fishman (ed.): Reading in the sociology of Language, The Hague, Mouton, 1968, pp. 139169.

HASBÚN, Leyla: The role of lexical aspect in the acquisition of the tenselaspect system in L2 Spanish, Indiana University, Tesis doctoral, 1995.

HILL, Sam y William BRADFORD: Bilingual grammar of English-Spanish syntax, London, Lanham, 1991.

HUSSEIN, Nada: Una propuesta de gramática pedagógica para aprendices de ELE árabo-parlantes basada en el análisis de errores del sistema verbal español, Universidad de Granada, Tesis doctoral, 2004.

LAFFORD, Barbara: "Spanish Applied Linguistics in the Twentieth Century: A Retrospective and Bibliography (1900-99)". Hispania, 83-4 (2000), pp. 711-732.

LAFFORD, Barbara: "The development of tense/aspect relations in L2 Spanish narratives: evidence to test competing theories". Paper read at SLRF 96. Tucson Az, 1996.

LEONTARIDI, Eleni: "Análisis Contrastivo del Aspecto Gramatical en Español y en Griego Moderno". En AAVV: Actas del I Congreso de estudiantes de Filología Hispánica / La palabra es futuro: filólogos del nuevo milenio, Valladolid, Universidad de Valladolid, 2002, pp. 129-144.

LEONTARIDI, Eleni: Los tiempos del pasado del indicativo en español y en griego moderno, Universidad de Salamanca, Tesis doctoral, 2001.

LUBBERS-QUESADA, Margaret: "L2 acquisition of the Spanish subjunctive mood and prototype schema development". Spanish Applied Linguistics, 2 (1998), pp. 1-23.

MANSILLA, Matilde: "Valores y usos del presente en inglés y en español". Senara, 2 (1980), pp. 113-50.

MAO, Pie-wen: "La aspectualidad en la interlengua de estudiantes taiwaneses de ELE. Un estudio empírico sobre la dicotomía pretérito indefinido/pretérito imperfecto". Revista Nebrija de Lingüistica Aplicada, 5-3 (2009), pp. 87-103

MARKIC, Jasmina: "El aspecto verbal como problema de traducción del español al esloveno y del esloveno al espanol." Vestnik. Filozofska fakulteta, Ljubljana, 34 (2000), pp.365-69.

MARKIC, Jasmina: "Hacia un estudio aspectual contrastivo entre el esloveno y el español." Verba Hispanica, 1 (1991), pp. 105-10.

MARTIN GONZÁLEZ Ma Rocío: El análisis de errores en español como lengua extranjera: el caso del checo, Universidad Autónoma de Madrid, Tesis doctoral, 1999.

MARTÍNEZ-ATIENZA, María: "El sistema tempo-aspectual del español, italiano e inglés: un análisis contrastivo". En M. Villayandre (ed.): Actas del XXXV Simposio 
de la Sociedad Española de Lingüistica, León, Universidad de León, 2006, pp. 1266-1288.

MEDINA, Mario: "Análisis comparativo del uso del subjuntivo en alemán y en español”. Beiträge zur romanischen Philologie, 18 (1979), pp. 267-271.

MONTRUL, Silvina y Roumyana SLABAKOVA: "The L2 Acquisition of Morphosyntactic and Semantic Properties of the Aspectual Tenses Preterite and Imperfect”. En A.T. Perez-Leroux y J. Liceras (eds.): The Acquisition of Spanish Morphosyntax, Dordrech, Kluwe, 1999, pp. 131-149.

MORENO, Concha y Gretel ERES: Gramática contrastiva del español para brasileños, Madrid, SGEL, 2007.

MuÑIZ, $\mathrm{M}^{\mathrm{a}}$ de las Nieves "Uso del condicional en italiano y español: estudio contrastivo". En J. Arce, M. Carrera, F. F. Murga y M. Muñiz (eds.): Italiano y Español. Estudios lingüísticos, Utrera, Universidad de Sevilla, 1984, pp. 127-157.

NARBONA, Antonio: Sintaxis española: nuevos y viejos enfoques, Barcelona, Ariel, 1989.

NORTH, Brian: The development of descriptors on scales of language proficiency, Washington DC, National Foreign Language Center, 1993.

RAMSEY, Violeta: Developmental stages in the acquisition of the perfective and the imperfective aspects by classroom L2 learners of Spanish, University of Oregon, Tesis doctoral, 1990.

RODRÍGUEZ OSUNA, Jacinto: "La muestra: teoría y aplicación". En M. García Ferrando, J. Ibáñez y F. Alvira (comps.): El análisis de la realidad social. Métodos y técnicas de investigación, Madrid, Alianza, 2003 pp.446-482.

RUIZ, Raúl. y Juan Ramón GUIJARRO: "Análisis contrastivo inglés-español: morfología, funcionalidad y semántica del imperfecto y del pretérito para estudiantes de español lengua extranjera". RedELE, 4 (2005).

SALABERRY, Rafael: "The development of past tense verbal morphology in classroom L2 Spanish. Applied Linguistics, 20-2 (1999), pp. 151-178.

SÁNCHEZ CASTRO, Marta: "Dificultades de los hablantes nativos de alemán en el aprendizaje de los tiempos de pasado". RedELE, 13 (2008).

SÁNCHEZ CASTRO, Marta: Contrastes. Español para hablantes de alemán, Madrid, SGEL, 2007.

SÁNCHEZ IGLESIAS, Jorge: Errores, corrección y fosilización en la didáctica de lenguas afines: análisis de errores en la expresión escrita de estudiantes italianos de E/LE, Salamanca, Ediciones Universidad de Salamanca, 2003.

SÁNCHEZ PRIETO, Raúl "Transferencias e interferencias lingüísticas en el uso de los tiempos verbales de indicativo en español y alemán". En V. González Martín (coord.): Hacia la unidad en la diversidad. Difusión de las lenguas europeas. Año europeo de las lenguas 2001, Salamanca, Diputación Provincial, 2003.

SÁNCHEZ PRIETO, Raúl: Estudio contrastivo de los tiempos de pasado en indicativo en español y alemán, Frankfurt/Main, Peter Lang, 2004.

SÁNCHEZ PRIETO, Raúl: Los usos modales en las formas verbales de indicativo: diferentes realizaciones en español y alemán". En AAVV: Germanística y 
enseñanza del alemán en España, Vol. 1: Alemán como Lengua Extranjera y Lingüística (coordinado por B. Balzer), Madrid, Editorial Idiomas, 2008.

SANTOS GARGALLO, Isabel: Análisis contrastivo, análisis de errores e interlengua en el marco de la Lingüistica contrastiva, Madrid, Síntesis, 1993.

SANTOS, Julián y otros: Diseño de encuestas para estudios de mercado. Técnicas de muestreo y análisis multivariante, Madrid, Centro de Estudios Ramón Areces, 2004.

SCHIFKO, Peter: Subjonctif und subjuntivo. Zum Gebrauch des Konjunktivs im Französischen und Spanischen, Wien, Stuttgart, W. Braumüller, 1967.

SOHAMY, Elana "The relationship between language testing and second language acquisition revisited". System, 28 (2000) pp. 541-553.

Solé, Yolanda. y Carlos. Solé: Modern Spanish Syntax. A study in contrast, Lexington-MA, DC Heath and Company, 1977.

Sossouvi Laurent: Análisis de errores de los alumnos benineses de español como lengua extranjera, Universidad de Alcalá de Henares, Tesis doctoral, 2003.

STOKES, Jeffrey: "Some factors in the acquisition of the present subjunctive in Spanish". Hispania, 7 (1988), pp. 705-710.

TORIJANO, Agustín: Análisis teórico-práctico de los errores gramaticales en el aprendizaje del español, L-2: expresión escrita, Salamanca, Ediciones Universidad de Salamanca, 2002.

TRESACO, M $\mathrm{M}^{\mathrm{a}}$ Pilar: Estudio contrastivo de las formas temporales del francés y del español, Universidad de Zaragoza, Tesis doctoral, 1982.

Whitley, M. Stanley: Spanish/English Contrasts, Washington DC, Georgetown University Press, 1986.

WU DE ZIERER, Carolina: El gerundio español. Sus equivalentes en los idiomas inglés, francés y alemán, Trujillo, Universidad Nacional de Trujillo, 1965.

ZAMORANO, Juan: La generación de tiempo y aspecto en inglés y español: un estudio funcional contrastivo, Universidad Complutense de Madrid, Tesis doctoral, 2006.

ZHOU, Minkang: Estudio comparativo del chino y el castellano en los aspectos lingüisticos y culturales, Universidad Autónoma de Barcelona, Tesis doctoral, 1995.

ZIMMER, Rudolph: Die Morphologie des italienischen, spanischen und portugiesischen Verbs. Einsprachlich und im Vergleich, Tübingen, Niemeyer, 1992. 